\title{
The SCUBA-2 Cluster Snapshot Survey - I. Catalogue of lensed galaxies and submillimetre-bright central galaxies
}

Ryan A. Cheale, ${ }^{1 \star}$ James E. Geach ${ }^{\oplus},{ }^{1}$ Alastair C. Edge, ${ }^{2}$ Andrew W. Blain, ${ }^{3}$
Scott C. Chapman, ${ }^{4}$ Eiichi Egami, ${ }^{5}$ Mike T. Hogan, ${ }^{2}$ Timothy D. Rawle ${ }^{7}$ and Tracy M. A. Webb ${ }^{6}$

${ }^{1}$ Center for Astrophysics Research, Science \& Technology Research Institute, University of Hertfordshire, Hatfield AL10 9AB, UK

${ }^{2}$ Centre for Extragalactic Astronomy, Department of Physics, Durham University, South Road, Durham DH1 3 LE, UK

${ }^{3}$ Physics \& Astronomy, University of Leicester, 1 University Road, Leicester LE1 7RH, UK

${ }^{4}$ California Institute of Technology, 1200 East California Boulevard, Pasadena, CA 91125, USA

${ }^{5}$ Steward Observatory, University of Arizona, 933 North Cherry Avenue, Tucson, AZ 85721, USA

${ }^{6}$ McGill Space Institute, Department of Physics, McGill University, 3600 rue University Montreal, Quebec, Canada, H3P $1 T 3$

${ }^{7}$ European Space Agency, c/o STScI, 3700 San Martin Drive, Baltimore, MD 21218, USA

Accepted 2018 December 12. Received 2018 November 23; in original form 2018 June 5

\section{ABSTRACT}

The SCUBA-2 Cluster Snapshot Survey (S2CSS) observed 202 rich clusters of galaxies at $850 \mu \mathrm{m}$ in relatively poor submillimetre observing conditions $\left(\tau_{225 \mathrm{GHz}}>0.1\right)$ with the aim of identifying rare examples of bright (tens of $\mathrm{mJy}$ ) gravitationally lensed submillimetre galaxies. The S2CSS covered over $0.33 \mathrm{deg}^{2}$ to an average depth of $\sigma_{850} \approx 12 \mathrm{mJy} \mathrm{beam}^{-1}$. Here we present a sample of 97 bright $850-\mu \mathrm{m}$ point sources selected from the S2CSS that are potentially gravitationally lensed, and eight sources for which the strong submillimetre emission is co-located with the central brightest cluster galaxy (BCG). We construct farinfrared spectral energy distributions for those sources with Herschel SPIRE coverage and use these distributions to estimate the redshifts and luminosities of the sources. The bright submillimetre flux density of our sources makes them excellent targets for detailed follow-up work that will allow the detection of spectral features in the submillimetre/millimetre that would otherwise be too faint to detect. Through a stacking analysis, we also investigate the average submillimetre/radio properties of BCGs, determining the average $850-\mu \mathrm{m}$ flux of BCGs as a function of radio luminosity.

Key words: galaxies: active-galaxies: clusters: general-galaxies: evolution-galaxies: high-redshift-cosmology: observations - submillimetre: galaxies.

\section{INTRODUCTION}

Twenty years ago, 'submillimetre galaxies' (SMGs) were discovered (Smail, Ivison \& Blain 1997; Hughes et al. 1998; Barger et al. 1998) with the Submillimetre Common User Bolometer Array (SCUBA; Gear \& Cunningham 1990; Holland et al. 1999). Optically faint (Hughes et al. 1998; Smail et al. 1999), SMGs lie out at $z \approx 1-4$ (see Chapman et al. 2005; Coppin et al. 2006; Aretxaga et al. 2007; Ivison et al. 2007; Dye et al. 2008; Serjeant et al. 2008; Simpson et al. 2014; Danielson et al. 2017) near the peak in the cosmic star formation rate density (SFRD) (Hopkins \& Beacom 2006; Madau \& Dickinson 2014). SMGs have infrared luminosities and intrinsic properties similar to those of local ultra-luminous infrared galaxies (ULIRGs), with SMGs having $L \approx 10^{12} \mathrm{~L} \odot$ (Houck et al. 1985;
Dey et al. 1999; Chapman et al. 2005), intense star formation rates of $10^{2}-10^{3} \mathrm{M}_{\odot} \mathrm{yr}^{-1}$ (Tacconi et al. 2010; Magnelli et al. 2012) and stellar masses of order $10^{10-11} \mathrm{M}_{\odot}$ (Hainline et al. 2011); however, the link between SMGs and ULIRGs is still not clear, given the size distributions of ULIRGs (e.g. Simpson et al. 2015; Spilker et al. 2016). SMGs are excellent candidates for the progenitor population of the massive elliptical galaxies seen today (see Lilly et al. 1999; Eales et al. 2000; Daddi et al. 2009; Simpson et al. 2014; Toft et al. 2014) and serve as a useful tool for probing the interstellar medium (ISM) and the crucial mechanisms at play in the evolution of dusty star-forming galaxies (Valtchanov et al. 2011; Danielson et al. 2011, 2013; Bothwell et al. 2013).

In the $850-\mu \mathrm{m}$ band, a flux-limited survey will return a nearconstant infrared luminosity selection between redshifts of 1 and 7, owing to the well-known negative $k$-correction, whereby cosmological dimming is cancelled out by the fact that a fixed 
observed bandpass will climb up the redshifted Rayleigh-Jeans tail of the thermal dust emission (Blain et al. 2002). Although the redshift distribution is strongly influenced by selection effects, for example the dust temperature plays a significant role (e.g. Béthermin et al. 2015), the bias can be quantified by increasing the number of SMGs detected at various redshifts. The negative $k$-correction makes submillimetre observations very efficient at detecting actively growing galaxies across most of cosmic history. However, the bright confusion limit (e.g. Blain \& Longair 1996) of ground-based submillimetre surveys typically limits SMG surveys to a few mJy (e.g. Karim et al. 2013). At these limits, detailed follow-up work with submillimetre/millimetre-band interferometry is challenging. Instruments such as the Atacama Large Millimeter/Submillimeter Array (ALMA) can obtain high-resolution data, but this requires brighter targets. Indeed, the ALMA era has already discovered spectacular examples of high-redshift SMGs out to redshift 7 (e.g. SPT0311-58, Marrone et al. 2018 and A1697-zD1, Knudsen et al. 2017).

Gravitational lensing (Schneider, Ehlers \& Falco 1992) allows the low-flux limit to be overcome by boosting the intrinsic flux density of distant galaxies that are fortuitously aligned with foreground masses such as other galaxies or clusters (Smail et al. 1997). By preferentially selecting cluster-galaxy systems as target lenses, the differential lensing bias can be minimized (Serjeant 2012), and the magnification of background galaxies allows detailed studies that would otherwise be impossible, yielding new insights into the properties of early galaxies (Ivison et al. 1998, 2010; Valtchanov et al. 2011; Combes et al. 2012; Decarli et al. 2012; Omont et al. 2013). Arguably, the most famous example of an extremely lensed galaxy is the 'Cosmic Eyelash' $\left(z=2.3259, S_{870} \approx 100 \mathrm{mJy}\right.$; Swinbank et al. 2010), a highly lensed SMG with a magnification of $32 \times$, boosting the $870-\mu \mathrm{m}$ flux density to $103 \mathrm{mJy}$. The Eyelash has provided a treasure trove of information, including insights into the properties of the cold and dense ISM (see Danielson et al. 2011, 2013; George et al. 2014), probing 10-pc giant molecular cloud complexes (Thomson et al. 2015), molecular outflows (George et al. 2014), and evidence arguing against the SMG-ULIRG scaling (Ivison et al. 2010).

Galaxies like the Eyelash are exceptionally rare. Gathering a larger sample of similarly bright lensed SMGs would be of immense value, providing target sources for detailed follow-up observations, especially with powerful interferometric facilities such as the NOrthern Extended Millimeter Array (NOEMA) and ALMA. This realization motivated the SCUBA-2 Cluster Snapshot Survey (S2CSS), described in this work, which was conducted with the SCUBA-2 submillimetre camera mounted on the 15-m James Clerk Maxwell Telescope (JCMT, Holland et al. 2013). Using the longer-wavelength capability of SCUBA-2, we can preferentially select lensed background sources over dusty member galaxies in the cluster. In contrast, the shorter-wavelength surveys with Herschel (e.g. Coppin et al. 2011) show a statistical excess of bright far-infrared (FIR) dusty cluster members. However, few cluster members can be detected at $850 \mu \mathrm{m}$ through their strong dust continuum or synchrotron emission that dominates the cluster emission in the short- and millimetre-wavelength regime (Edge et al. 1999; Hogan et al. 2015). Even in poor submillimetre weather conditions $\left(\tau_{225 \mathrm{GHz}} \geq 0.12\right)$, point sources with flux densities of order $100 \mathrm{mJy}$ can be detected with high signal-to-noise ratios in relatively short integration times. By narrowing the survey to targets where one might expect to find lensed background sources (massive clusters of galaxies), and conducting observations in poor conditions, we can systematically hunt for previously unknown strongly lensed, Eyelash-like SMGs.

This paper is organized as follows. In Section 2 we describe the target selection, observations and data reduction. Section 3 discusses the process of determining the nature of the sources. Section 4 provides an analysis on the brightest cluster galaxy (BCG) properties over multiple wavebands. Section 5 discusses the sources detected and the nature of the BCG stacking analysis. Finally, Section 6 summarizes the results and details future work. Throughout we assume a $\Lambda \mathrm{CDM}$ cosmology with $H_{0}=67.3 \mathrm{~km}$ $\mathrm{s}^{-1} \mathrm{Mpc}^{-1}, \Omega_{\mathrm{M}}=0.315$, and $\Omega_{\Lambda}=0.685$ (Planck Collaboration et al. 2014).

\section{SURVEY STRATEGY, OBSERVATIONS AND DATA REDUCTION}

Here we describe our survey targets, selection criteria and how we reduced the data.

\subsection{Target selection}

Our targets are rich clusters of galaxies visible by the JCMT. These are the most massive targets that we could choose, and we therebymaximize the probability of detecting lensed background sources because the SCUBA-2 field of view (FOV) $\left(45 \operatorname{arcmin}^{2}\right)$ exceeds the Einstein radius $\left(r_{\mathrm{E}} \approx 20 \mathrm{arcsec}\right)$ for a typical clusterlens system (properties of order: $M_{\text {cluster }} \approx 10^{14} \mathrm{M}_{\odot}, \mathrm{z}_{\text {cluster }} \approx$ $0.5, z_{\text {source }} \approx 2.5$ ). Specifically, we target clusters with the highest X-ray luminosities $\left(L_{\mathrm{X}} \geq 4 \times 10^{44} \mathrm{erg} \mathrm{s}^{-1}, 0.1-2.4 \mathrm{keV}\right)$. The clusters were selected from the ROSAT All-Sky Survey (Voges et al. 1999), BCS (Ebeling et al. 1998), eBCS (Ebeling et al. 2000), REFLEX (Böhringer et al. 2004) and MACS (MACS; Ebeling, Edge \& Henry 2001; Ebeling et al. 2013) samples, which constitute the most X-ray-luminous, and therefore the most massive, clusters in the local Universe $(z \approx 0.5)$. The majority of the sample objects have matching Herschel SPIRE data obtained from the Herschel Lensing Survey (HLS; Egami et al. 2010; Egami et al., in preparation). In particular, many of the S2CSS-targeted clusters were observed in the HLS-Snapshot I Survey, which obtained shallow but nearly confusion-limited SPIRE images for 279 X-ray-luminous clusters. The majority of the sample matches the cluster selection for the Herschel Lensing Survey Snapshot-1 sample, comprising 279 clusters from the X-ray samples that had not already been scheduled for Herschel in the first two cycles. Of these clusters, 216 are visible from Mauna Kea $\left(\delta \geq-30^{\circ}\right)$, and 202 were successfully observed in the S2CSS. Of the 72 remaining X-ray-selected clusters, a total of 48 have Herschel data from a combination of projects: Herschel Lensing Survey Deep-1, LoCUSS and the Cooling Flow Open Time Key Project (Edge et al. 2010; Egami et al. 2010; Smith et al. 2010; Rawle et al. 2012). An extra nine clusters with lower X-ray luminosity and particularly radio-bright central galaxies were also included, and the results for these are summarized in Hogan et al. (2015).

\subsection{Observations and data reduction}

Observations were conducted in the JCMT weather bands 4 and 5 over the period from 2012 August to 2013 July (formally, a scheduling limit of $\tau_{225 \mathrm{GHz}}>0.12$ ), corresponding to a precipitable water vapour column of over $2.6 \mathrm{~mm}$. The top panel of Fig. 1 shows the distribution of $\tau_{225 \mathrm{GHz}}$ for the observations; the average opacity is $\left\langle\tau_{225 \mathrm{GHz}}\right\rangle=0.19 \pm 0.08$. A small number of maps taken in 

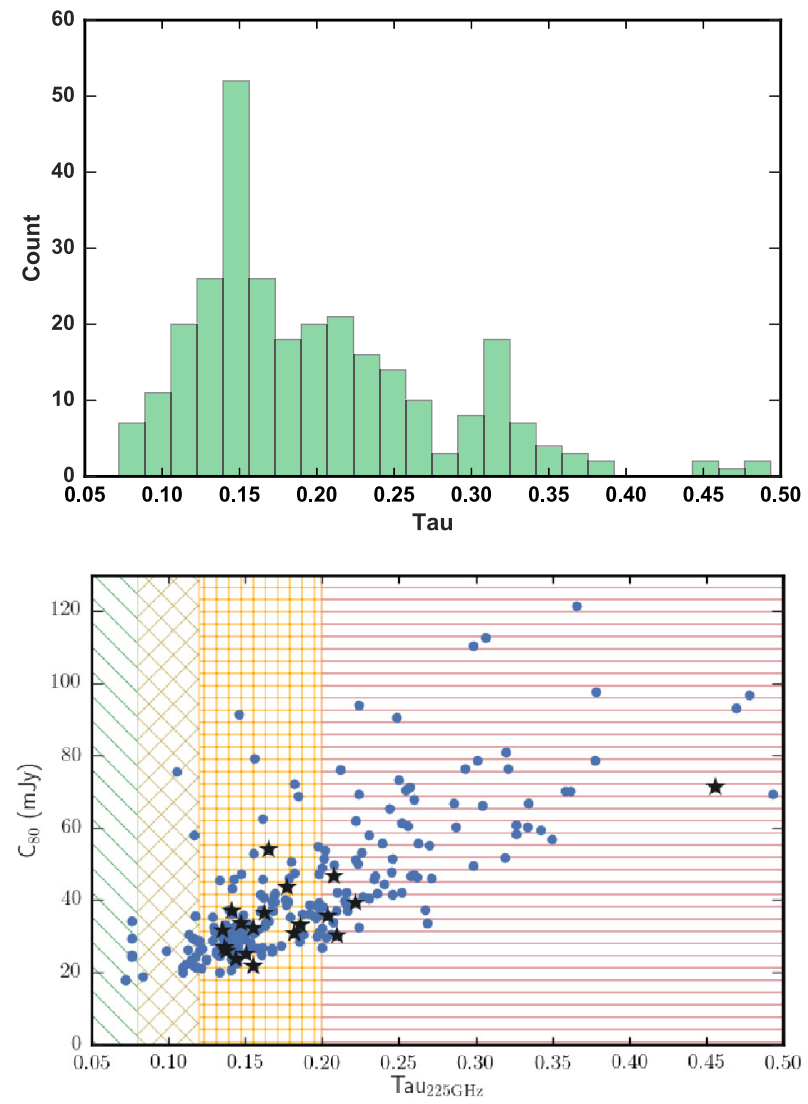

Figure 1. (Top) The S2CSS $\tau_{225 \mathrm{GHz}}$ distributions for all observations. The observations were conducted primarily in JCMT band 4 weather conditions $\left(\tau_{225 \mathrm{GHz}}=0.19 \pm 0.8\right)$, corresponding to a 53 per cent extinction. (Bottom) The plot of $\tau_{225 \mathrm{GHz}}$ versus $C_{80}$ of the sample shows a clear relationship between the observing weather conditions and the completeness of each map. Black stars indicate sources with significant SPIRE counterparts, the different hatched regions represent the JCMT weather banding (1-4) in increasing ranges of precipitable water vapour.

better conditions than requested but with a longer tail of higher $\tau_{225 \mathrm{GHz}}$ values were also recorded. We adopted the 'CV_DAISY' mapping pattern to maximize the exposure time on cluster centres (Thomas \& Currie 2014), with a beam size of 13 arcsec. The camera was slewed in a Lissajous-like pattern at constant velocity, ensuring that the target remained on the SCUBA-2 array throughout the 30min observation (e.g. Geach et al. 2013, hereafter G13). Data at $450 \mu \mathrm{m}$ were recorded simultaneously, but the conditions were too poor for the data to be scientifically useful in all cases.

Data were reduced using the dynamic iterative map maker (DIMM) of the SubMillimetre User Reduction Facility (SMURF; Chapin et al. 2013). We followed the same data reduction procedure as described in G13, which accounts for flat-fielding, common mode subtraction, extinction correction, and aggressive spatial filtering (because we are interested only in point sources). The maps were all match-filtered to maximize the sensitivity to the detection of point sources. We applied a constant flux conversion factor, $\mathrm{FCF}_{850}=556 \pm 65 \mathrm{Jy}$ beam ${ }^{-1} \mathrm{pW}^{-1}$, with an additional 10 percent correction to account for flux lost during the filtering process (determined by inserting a bright Gaussian point source into the time stream of each individual observation to measure the model response to the filtering, as in G13). The total survey area is approximately a third of a square degree, with a median depth per cluster field of $\sigma_{850}=12 \pm 6 \mathrm{mJy}$ beam $^{-1}$, with the error being the standard deviation of the $1 \sigma$ noise per field.

\section{$2.3850-\mu \mathrm{m}$ source detection and selection}

To detect point sources we simply used a peak-finding algorithm, with a signal-to-noise (SNR) threshold, on each observation; the local noise is determined from the instrumental noise map produced as part of the reduction process (the depth of the maps is far from confused, so we ignore confusion noise). Following this method, we detected 1016 sources with SNR $3.5 \leq \sigma<4$, 312 with $4 \leq \sigma<5$ and 39 with $\geq 5 \sigma$ across all observations. We opted to use the $351>4 \sigma$ detections as our initial candidate source list, as the contamination rate from false positives is non-negligible (e.g. Geach et al. 2017). Completeness simulations provide an independent check of the significance of the detection. We injected multiple point sources into a map and re-ran the detection algorithm to re-extract our fake sources. By repeating this numerous times over a number of flux intervals initiated at $100 \mathrm{mJy}$, we built completeness statistics for each map. We refer to the 80 percent completeness value for each map using the descriptor ' $C_{80}$ ' as a proxy for a $5 \sigma$ detection, as discussed, because a map-dependent measurement takes into account the error properties unique to each map and indicates its depth; that is, a low $C_{80}$ corresponds to a deeper and therefore more sensitive map. The bottom panel in Fig. 1 compares the calculated $C_{80}$ value for each observation with the atmospheric opacity $\left(\tau_{225 \mathrm{GHz}}\right)$ and provides an indicator of map sensitivity averaged over the full area covered. Source with significant SPIRE counterparts are denoted as black stars, with all except one observed in reasonable conditions. The top panel of Fig. 2 shows the distribution of $S_{850}$ versus $C_{80}$ for all our detected peaks (we show sources of $>3.5 \sigma$ to illustrate the sheer number of low- $\sigma$ detections compared with the 351 sources $>4 \sigma$ ); the black stars represent sources having bright $(>3 \sigma)$ SPIRE counterparts. It is clear that sources detected with a low $S_{850}$ flux density correspond to maps with a low $C_{80}$ value for sources detected at $>3.5 \sigma$

We compared the median depth of all maps to the median 80 per cent completeness, confirming that a $5 \sigma$ limit corresponds to approximately the $C_{80}$ value (e.g. Saha 1995) in each map, allowing us to slightly tailor our selection criteria by jointly using the $1 \sigma$ instrumental noise and the completeness curve. We opted for this method over simply using the $5 \sigma$ map depth because the majority of the data were taken in poor JCMT weather bands and a mapdependent descriptor of the sensitivity is a better measure than using a sweeping $5 \sigma$ limit. We also found that the noise in each map sharply increases at larger radii when taking annular rings from the centre. Given the noise profile of the SCUBA-2 maps we applied an additional radial component to our selection to take this into account. For selection, we employ a hand-crafted selection criterion for the S2CSS catalogue that uses SCUBA-2 photometry, the radius $(R)$ from the map pointing centre (usually the position of the BCG), and the ratio of the detection flux to the $C_{80}$ value, $S_{850} / C_{80}$, for each observations. By combining all the factors discussed, we insist that the nature of a selected source exceeds

$\frac{S_{850}}{C_{80}} \geq 0.6+0.05 R^{2}$,

which is illustrated in the bottom panel of Fig. 2, panel 2, as a solid black line. We used a hard radial cut-off at 3.5 arcmin from the pointing centre, which covers the deepest region of the SCUBA-2 DAISY mapping pattern. With our layout, we determined 106 lens candidates across the survey, with a mean flux density 

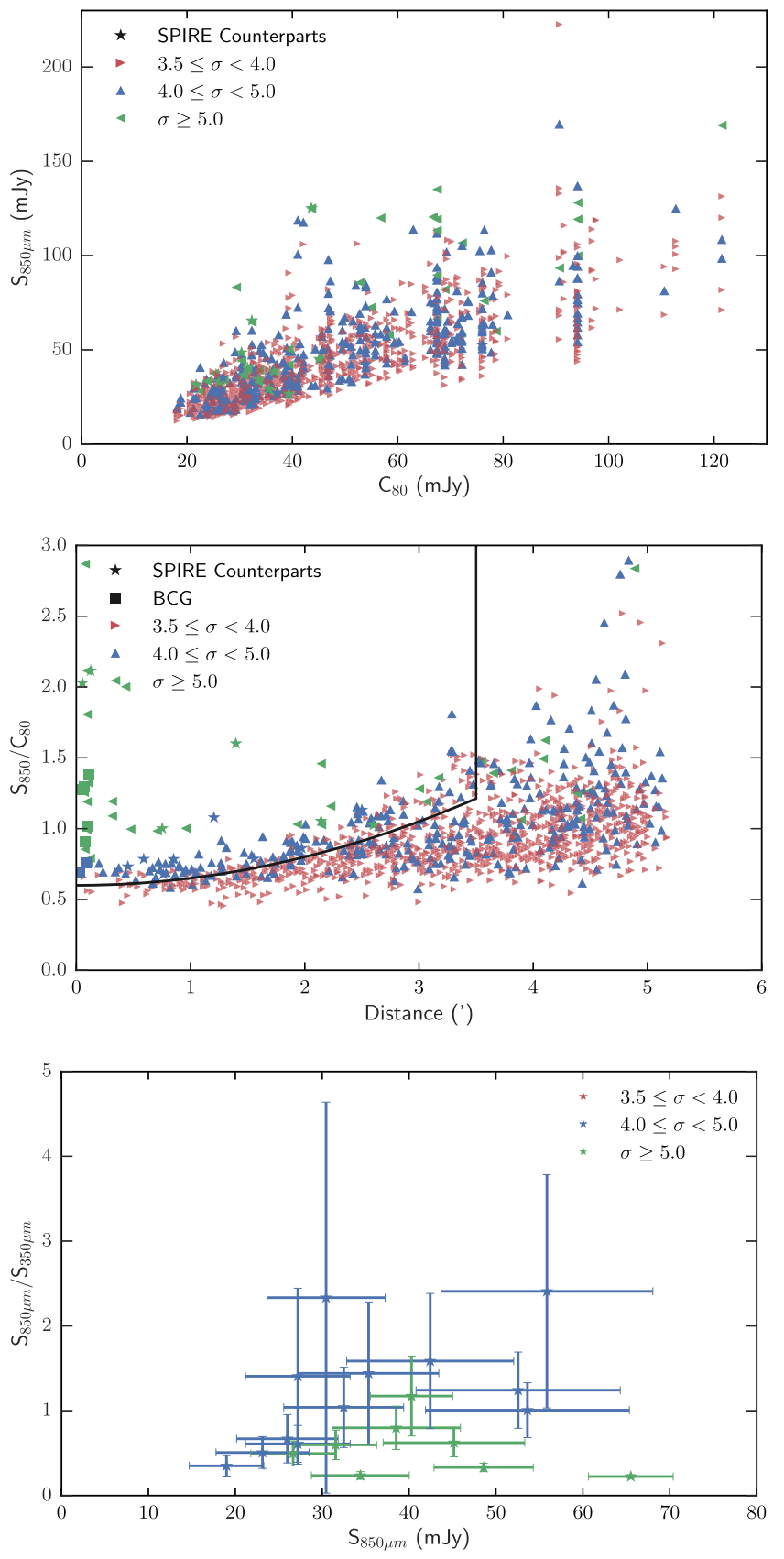

Figure 2. (Top) The peak flux density of the detection against the 80 per cent completeness of the map. It is evident that a large number of sources correlate with a low flux density and a corresponding low $C_{80}$. The seemingly vertical columns are a result of a single map showing a considerable number of detections. The SPIRE counterparts are stars that are colour-coded with respect to $\sigma$. (Middle) The ratio $S_{850} / C_{80}$ plotted as a function of radial distance from the SCUBA-2 field centre. The ratio of $S_{850} / C_{80}$ is related to the signal-to-noise ratio and provides a clear metric for false positives in our sample. We truncate our search at $3.5 \sigma$ to cover the deepest region of the SCUBA-2 DAISY mapping pattern. The sources with signifcant SPIRE counterparts are filled stars and the brightest cluster galaxies detected are filled squares, with both colour-coded with respect to $\sigma$. (Bottom) A high-confidence sample of our cross-matched HLS sources represented as a $850 \mu \mathrm{m} / 350 \mu \mathrm{m}$ to $850 \mu \mathrm{m}$ colour-colour plot. of $\left\langle S_{850}\right\rangle=43 \mathrm{mJy}$. They are listed in Table A1. The expected surface density of sources of similar flux density in blank fields is $N\left(S_{850 \mu \mathrm{m}}>15 \mathrm{mJy}\right)\left(\mathrm{deg}^{-2}\right) \approx 2$ (see Geach et al. 2017$)$, whereas this work finds $N\left(S_{850 \mu \mathrm{m}}>15 \mathrm{mJy}\right)\left(\mathrm{deg}^{-2}\right) \approx 300$. Hence, by biasing observations towards massive clusters we have successfully detected sources well beyond the limitations of a blank-field survey. Sources with a formal $\geq 5 \sigma$ detection at the pointing centre will of course be preferentially detected: these are cases where the submillimetre emission originates from the BCG itself, or from a strongly lensed background source along the same line of sight.

\subsection{Herschel Lensing Survey data products}

The Herschel Lensing Survey (HLS) consists of two types of surveys: HLS-Deep, which conducted deep PACS $(100 / 160 \mu \mathrm{m})$ and SPIRE $(250 / 350 / 500 \mu \mathrm{m})$ imaging for 54 clusters, and HLSSnapshot, which carried out shallower SPIRE-only imaging for 527 clusters (E. Egami et al., in preparation). Many of the S2CSS target clusters were selected from the latter sample, whose SPIRE images are nearly confusion-limited despite their short integration times. For all clusters, images were produced via the standard reduction pipeline in HIPE (Ott 2010) ver. 12 (ver. 12.2 calibration product), with median baseline removal and using the destriper. All the observations target the nominal cluster centre and have a beam size of 18,25 and $36 \operatorname{arcsec}$ respectively. The S2CSS and HLS complement one another, and in cases where there are joint SCUBA-2/SPIRE detections the data sets provide greater confidence in the nature of detections and allow us to roughly estimate redshifts through FIR colours: a $850 \mu \mathrm{m} / 350 \mu \mathrm{m}$ to $850 \mu \mathrm{m}$ colour-colour plot is given in the bottom panel of Fig. 2

\section{ANALYSIS}

In the following, we examine the 108 sources to determine whether they are lensed background sources, or cases where strong submillimetre emission originates from the BCG. We find that eight are co-located with the BCG, 78 are $>0.1$-arcmin SCUBA2-only detections, 19 are joint SCUBA-2 and SPIRE sources, and three were targeted in order to follow up a known SPIRE source.

\subsection{Nature of the SCUBA-2 submillimetre sources}

From the pool of 108 SCUBA-2 sources that fit the criteria described above, we found that 78 (non-starred in Table A1) have no significant $(<3 \sigma)$ SPIRE counterpart or are $<0.1$ arcmin from the cluster BCG, 19 have SPIRE counterparts, and three were targeted in order to follow up a known SPIRE source (see Appendix D). The cluster BCG was taken as the brightest cluster member nearest the X-ray peak (see Sanderson, Edge \& Smith 2009). In Fig. C1, we show all the S2CSS detections overlaid as contours on PanSTARRS $i$-band optical imaging (see Flewelling et al. 2016) to show the location of the submillimetre sources relative to the cluster centres/BCGs.

For eight of the sources, the Pan-STARRS $i$-band imaging (Fig. 3) listed in Table 1, the submillimetre contours are co-located with the position of the BCG, and indeed our precursor work (Hogan et al. 2015, H15) showed that these fields contain BCGs with strong 

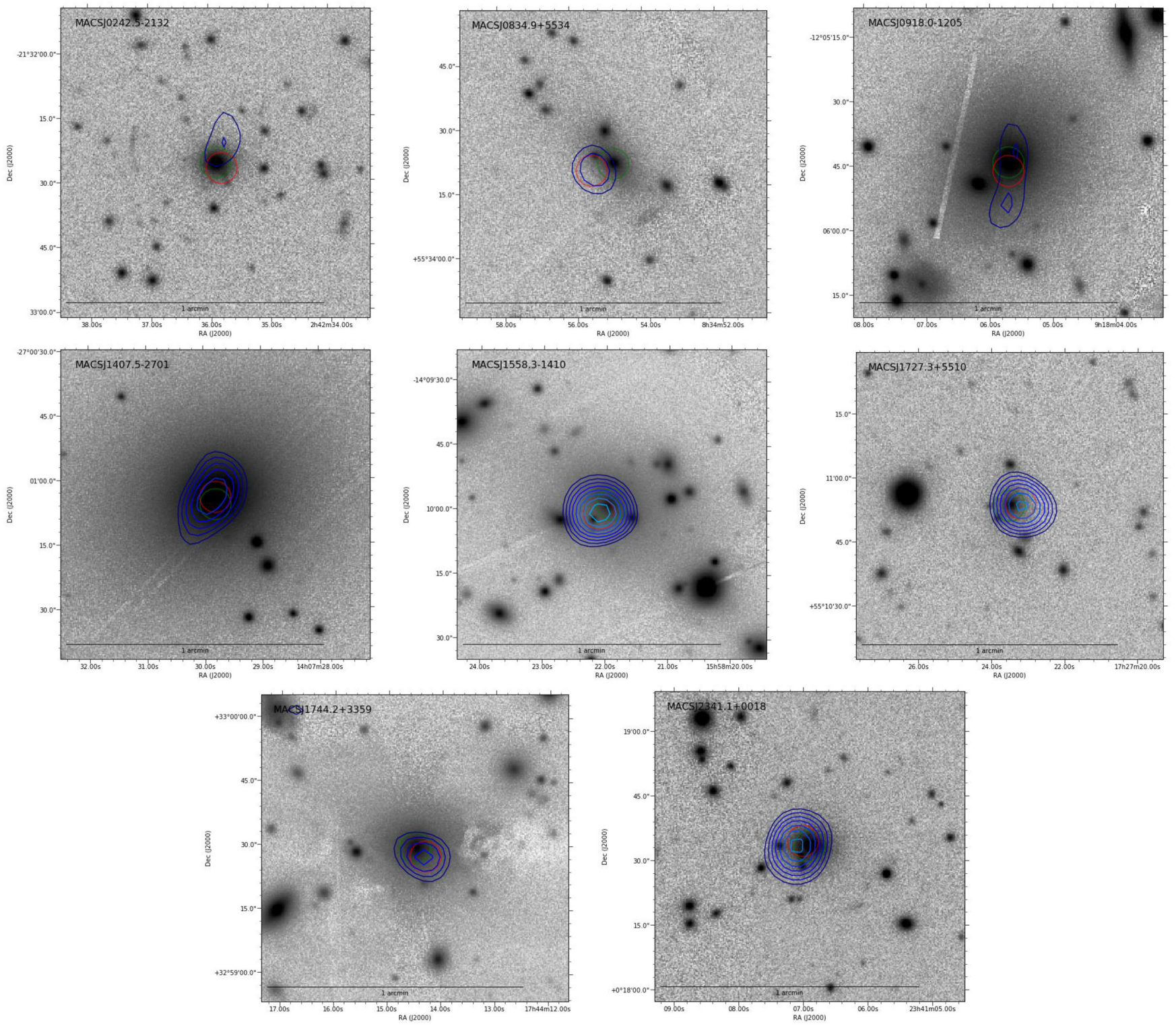

Figure 3. Pan-STARRS $i$-band imaging, with SCUBA-2 contours overlaid ( $3 \sigma$ in $0.5 \sigma$ increments), of the eight BCG sources found in S2CSS and H15. Red circles, BCGs; green circles, detections. Left to right by row: MACSJ0242.5-2132, MACSJ0834.9+5534, MACSJ0918.0-1205, MACSJ1407.5-2701, MACSJ1558.3-1410, MACSJ1727.3+5510-B, MACSJ1744.2+3259 and MACSJ2341.1+0018-A fields.

Table 1. Brightest cluster galaxy sources. GISMO/CARMA/AMI data from H15; observations closest in time to SCUBA-2 quoted. Column 1, source identification; column 2, cluster name; column 3, SCUBA-2 850- $\mu$ m peak flux; column 4, GISMO 150-GHz peak flux; column 5, CARMA 90-GHz peak flux; column 6, AMI 16-GHz peak flux.

\begin{tabular}{lccccc}
\hline ID & Cluster & $\begin{array}{c}S_{850 \mu \mathrm{m}} \\
{[\mathrm{mJy}]}\end{array}$ & $\begin{array}{c}S_{150 \mathrm{GHz}} \\
{[\mathrm{mJy}]}\end{array}$ & $\begin{array}{c}S_{90 \mathrm{GHz}} \\
{[\mathrm{mJy}]}\end{array}$ & $\begin{array}{c}S_{16 \mathrm{GHz}} \\
{[\mathrm{mJy}]}\end{array}$ \\
\hline MACSJ0242.5-2132 & MACS0242-21 & $34 \pm 8$ & $35.6 \pm 6.0$ & - & - \\
MACSJ0834.9+5534 & 4C+55.16 & $29 \pm 7$ & $81.7 \pm 13.2$ & - & $1317.7 \pm 66.0$ \\
MACSJ0918.0-1205 & Hydra-A & $76 \pm 9$ & $273.8 \pm 43.8$ & - & - \\
MACSJ1407.5-2701 & A3581 & $59 \pm 9$ & $62.1 \pm 10.1$ & $82.5 \pm 9.1$ & - \\
MACSJ1558.3-1410 & RXJ1558-14 & $40 \pm 4$ & $39.2 \pm 6.4$ & $44.8 \pm 4.9$ & - \\
MACSJ1727.3+5510-B & A2270 & $34 \pm 4$ & $70.8 \pm 4.1$ & $164.7 \pm 17.2$ & $226.9 \pm 11.6$ \\
MACSJ1744.2+3259 & Z8276 & $37 \pm 5$ & $12.5 \pm 2.7$ & $35.4 \pm 6.0$ & $87.6 \pm 4.6$ \\
MACSJ2341.1+0018-A & RXJ2341+00 & $33 \pm 3$ & $41.8 \pm 6.9$ & $72.8 \pm 8.0$ & $209.2 \pm 11.8$ \\
\hline
\end{tabular}


radio cores. In Table 1 , we also include multiband data spanning from $850 \mu \mathrm{m}$ to $16 \mathrm{GHz}$. In a recent ACT-POL survey, Datta et al. (2018) observed MACSJ2341 + 0018-A, reporting a 148$\mathrm{GHz}$ flux density of $S_{148 \mathrm{GHz}}=45.8 \pm 7 \mathrm{mJy}$, which agrees with our $150-\mathrm{GHz}$ observation reported in $\mathrm{H} 15$. We also reported, in $\mathrm{H} 15$, that there is $850-\mu \mathrm{m}$ emission co-located with the BCG in MACSJ0439.0 +0520 . Because the point source is $<4 \sigma$ we do not include it in our sample; however, we do find a $>4 \sigma$ source 2 arcsec from the BCG in the cluster (reported in Table A1). H15 reported no SCUBA-2 flux for both MACSJ2153.6 + 1741 (A2390) and MACSJ2325.3-1207 (A2597). We found SCUBA2 fluxes of $20.9 \pm 6.4$ and $25.4 \pm 7.2$ co-located at the BCG respectively, although we too do not formally accept these as sources for the S2CSS as they are $<4 \sigma$. Their fluxes do constrain the GPS model of Orienti \& Dallacasa (2014) further though, and MACSJ2325.3-1207 (A2597) is also reported in Tremblay et al. (2018). Five of the detected BCGs are in clusters with a lower X-ray luminosity than our main sample selection, so the fraction of submillimetre-bright BCGs in any mass-selected sample is particularly low, with only three found in over 200 observed clusters.

Interestingly, in MACSJ1348.8+2635 (A1795), another BCG emitter, we find a bright ( $30 \mathrm{mJy}, 4.5 \sigma)$ submillimetre source 0.5 arcmin from the position reported in H15. Either it is co-located with the BCG and is a coordinate error or it is a significantly bright lensed source located very close to the BCG. MACSJ0918.0-1205 (Hydra-A/3C 218) is the only detection (of all 106 sources) showing a double-lobe submillimetre morphology (Fig. 3, right panel of first row). This is a well-studied powerful radio galaxy, and its radio properties are discussed in detail in H15.

The 78 non-SPIRE detections are significantly offset $(>0.1$ arcmin) from their cluster BCG, have bright submillimetre flux densities that argue against them being cluster members (because their corresponding FIR luminosities would be unfeasibly high), and lack strong radio emission. They are therefore more likely to be background galaxies that have been lensed by a combination of the overall cluster potential plus a foreground cluster member rather than a radio-bright $\mathrm{AGN}$ or cluster member. Indeed, one of our detections is the Cosmic Eyelash (MACSJ2135.2-01012) with $S_{850 \mu \mathrm{m}}=120 \pm 9 \mathrm{mJy}$ (in agreement with Swinbank et al. 2010), which was included in the sample to ensure that we recovered this well-known source. We can further classify and investigate the properties of these bright submillimetre sources by examining additional data across the radio and submillimetre bands where they are available. The 19 sources that also have SPIRE detections are convincing examples of lensed cases having significant detections in all three SPIRE bands and no reported 1-100 GHz data (H15 targets the same clusters). All 97 detections form our catalogue of lens sources and are reported in Table A1, in which the SPIRE sources are marked by a superscript ' $H$ '; their SPIRE fluxes are reported in Table 2.

\subsection{Herschel SPIRE imaging: far-infrared spectral energy distribution}

In cases where we have joint SCUBA-2/SPIRE detections, we can construct FIR spectral energy distributions (SEDs). We extracted SPIRE photometry from the position of the SCUBA-2 source, estimating the uncertainties (including confusion noise, e.g. Nguyen et al. 2010; Hardcastle et al. 2013) by randomly sampling a large number of pixels around the source positions and evaluating the standard deviation of the flux distribution for the 22 sources and their host fields listed in Table 2. Of these sources, 18 are offset from the BCG target, three are targeted (discussed in Appendix D) and one is the Cosmic Eyelash.

We then performed a FIR photometric redshift fit to the photometry at $250-850 \mu \mathrm{m}$. We assume that the SED can be described by a greybody with emissivity term $\beta=1.5$ (Hildebrand 1983; Rawle et al. 2012):

$S(v) \propto\left[1-\mathrm{e}^{-\tau(v)}\right] B_{v}(T)=\frac{\left[1-\mathrm{e}^{-\tau(v)}\right] v^{3}}{\mathrm{e}^{h v / k T}-1}$.

$\tau(v)$ accounts for the optical depth, where $\tau(v)=\left(v / v_{0}\right)^{\beta}$ and $v_{0}$ is the frequency at which the optical depth tends to unity (i.e. $\sim 3 \mathrm{THz}$; Draine 2006; Conley et al. 2011).

We fitted the photometry with the redshift as a free parameter using least-squares fitting SCIPY packages, restricting the temperatures to values between 30 and $50 \mathrm{~K}$. We also evaluated the redshift for a fixed standard $40 \mathrm{~K}$ dust temperature. Table 2 gives the redshift estimates for the full temperature range and for the fixed $40 \mathrm{~K} \mathrm{fit}$, and Fig. D1 shows the best-fitting SEDs. We find that the FIR colours are consistent with high- $z$ sources behind the clusters, with redshifts in the range $z \approx 2-4$.

\subsection{AzTEC and RSR follow-up}

In a precursor paper, Zavala et al. 2015 (Z15) presented Large Millimeter Telescope (LMT) follow-up of four of the S2CSS sources reported here. Z15 reported on follow-up observations with AzTEC and RSR on the LMT of MACSJ0455.2+0657, MACSJ1022.4+5006, MACSJ1731.6+2252 and MACSJ2043.22144. The analysis in Z15 found CO line detections in MACSJ0455, MACSJ1731 and MACSJ2043, reporting redshifts of $z=2.927$, 2.712 and a blended source of $z=2.04,3.25,4.68$ respectively. In comparison, we found photometric redshifts for MACSJ0455 of $z=1.3-2.3$, for MACSJ1022 of $z=2.6-4.2$, for MACSJ1731 of $z=1.9-3.2$, and for MACSJ2043 of $z=1.8-31$. Thus, our photometric redshift estimates are at least reliable enough to determine that the remaining detections lie at high redshift behind the clusters.

Z15 used a conservative threshold for the signal-to-noise ratio of $\mathrm{S} / \mathrm{N} \geq 3.5$ to determine a $\mathrm{CO}$ transition line detection. They reported new 1.1-mm fluxes for three sources, which are also observed in this work: MACSJ1022 with $S_{1.1 \mathrm{~mm}}=11 \pm 2 \mathrm{mJy}$, MACSJ0455 with $S_{1.1 \mathrm{~mm}}=23 \pm 3 \mathrm{mJy}$, and MACSJ2043 with $S_{1.1 \mathrm{~mm}}=29 \pm 3 \mathrm{mJy}$. RSR observations found no detections for MACSJ1022, but this galaxy is one of the faintest in both Z15 and this work so it is likely that longer integration times are required. Photometric redshift-fitting in this work yields $2.6<z<4.2$, which is the typical expected range for a lensed submillimetre galaxy. MACSJ0455 has a single line detection, namelyv = $88.051 \mathrm{GHz}$ with $\mathrm{S} / \mathrm{N}=7.3$, and therefore we can provide a tentative redshift. Z15 suggested that the line is associated with the $\mathrm{CO}(2-1)$ transition, implying $z=1.618$; however, one would expect redshifts typically in the range $z=2-3$ for a lensed galaxy. Z15 deduced that the $\mathrm{CO}(3-2)$ transition is a more likely candidate at $z=2.927$, and this is in agreement with the redshift range found here of $1.8<z<3.0$. Multiple lines are detected in MACSJ2043, and this coincides with the image having the brightest $1.1-\mathrm{mm}$ detection. This work finds a wide range of redshift photometric fits of $1.8<z<3.1$; however, this does not account for the RSR detections of multiple lines, which raises the interesting possibility that they may originate from multiple sources. Four lines were detected at $v=75.827,81.178,101.459$ and $108.428 \mathrm{GHz}$ with 
Table 2. SPIRE photometry for the S2CSS sources and far-infrared photometric redshift estimates based on greybody fits to the SPIRE+SCUBA-2 photometry. The penultimate column indicates the range of acceptable redshifts for the range of dust temperatures 30-50 K, and the final column gives the redshift for a fixed temperature of $40 \mathrm{~K}$. The superscript ' $i$ ' denotes results obtained from Ivison et al. (2010), ' $T$ ' refers to the targeted sources in Appendix D, and ' $C E$ ' refers to the Cosmic Eyelash.

\begin{tabular}{|c|c|c|c|c|c|c|c|c|}
\hline ID & $\begin{array}{c}\text { SCUBA-2 position } \\
\text { [J2000] }\end{array}$ & $\begin{array}{c}C_{80} \\
{[\mathrm{mJy}]}\end{array}$ & $\begin{array}{c}S_{850} \\
{[\mathrm{mJy}]}\end{array}$ & $\begin{array}{c}S_{500} \\
{[\mathrm{mJy}]}\end{array}$ & $\begin{array}{c}S_{350} \\
{[\mathrm{mJy}]}\end{array}$ & $\begin{array}{c}S_{250} \\
{[\mathrm{mJy}]}\end{array}$ & $\begin{array}{l}\text { Photometric } \\
\text { redshift }\end{array}$ & $\begin{array}{c}T=40 \mathrm{~K} \\
\text { redshift }\end{array}$ \\
\hline MACSJ0417.5-1154 & 04:17:33.4-11:55:02.0 & 33 & $25 \pm 5$ & $62 \pm 15$ & $38 \pm 14$ & $54 \pm 13$ & $2.3-3.8$ & $3.1 \pm 0.3$ \\
\hline MACSJ0429.6-0253 & $04: 29: 33.3-02: 52: 35.5$ & 71 & $55 \pm 12$ & $32 \pm 15$ & $23 \pm 12$ & $32 \pm 12$ & $4.5-7.1$ & $5.8 \pm 0.8$ \\
\hline MACSJ0958.3-1103-B & 09:58:14.5-11:03:08.0 & 26 & $23 \pm 5$ & $27 \pm 17$ & $45 \pm 13$ & $35 \pm 13$ & $2.4-3.9$ & $3.2 \pm 0.4$ \\
\hline MACSJ0958.3-1103-C & 09:58:10.1-11:02:10.0 & 26 & $35 \pm 8$ & $14 \pm 17$ & $24 \pm 13$ & $23 \pm 13$ & $4.0-6.4$ & $5.3 \pm 0.9$ \\
\hline MACSJ1022.4+5006 & $10: 22: 26.6+50: 05: 38.5$ & 45 & $45 \pm 8$ & $76 \pm 17$ & $72 \pm 14$ & $57 \pm 14$ & $2.6-4.2$ & $3.4 \pm 0.3$ \\
\hline MACSJ1023.8-2715 & $10: 23: 57.1-27: 16: 53.0$ & 36 & $38 \pm 7$ & $55 \pm 18$ & $48 \pm 12$ & $23 \pm 13$ & $3.1-5.0$ & $4.1 \pm 0.4$ \\
\hline MACSJ1314.3-2515 & $13: 14: 21.2-25: 15: 47.5$ & 33 & $34 \pm 5$ & $106 \pm 17$ & $145 \pm 14$ & $136 \pm 14$ & $1.6-2.8$ & $2.3 \pm 0.2$ \\
\hline MACSJ1325.1-2013-B & $13: 24: 59.6-20: 13: 32.5$ & 53 & $42 \pm 9$ & $45 \pm 16$ & $26 \pm 11$ & $33 \pm 13$ & $3.6-5.8$ & $4.8 \pm 0.6$ \\
\hline MACSJ1722.4+3207-A & $17: 22: 30.2+32: 07: 32.0$ & 39 & $26 \pm 4$ & $36 \pm 15$ & $53 \pm 12$ & $50 \pm 13$ & $2.2-3.7$ & $3.0 \pm 0.3$ \\
\hline MACSJ1731.6+2252-A & $17: 31: 40.5+22: 50: 35.0$ & 30 & $48 \pm 5$ & $153 \pm 16$ & $146 \pm 13$ & $130 \pm 13$ & $1.9-3.2$ & $2.5 \pm 0.1$ \\
\hline MACSJ1910.4+6741 & $19: 10: 26.2+67: 41: 51.2$ & 37 & $27 \pm 6$ & $34 \pm 15$ & $19 \pm 13$ & $15 \pm 13$ & $3.6-5.8$ & $4.7 \pm 0.8$ \\
\hline MACSJ2043.2-2144 & $20: 43: 14.5-21: 44: 39.0$ & 32 & $65 \pm 4$ & $234 \pm 21$ & $292 \pm 15$ & $197 \pm 14$ & $1.8-3.1$ & $2.5 \pm 0.1$ \\
\hline MACSJ0326.8-0043 ${ }^{T}$ & $03: 26: 57.8-00: 45: 17.0$ & 35 & $29 \pm 5$ & $73 \pm 15$ & $72 \pm 13$ & $61 \pm 13$ & $2.1-3.6$ & $2.9 \pm 0.3$ \\
\hline MACSJ0455.2+0657 $7^{T}$ & $04: 55: 18.1+07: 01: 01.0$ & 30 & $36 \pm 5$ & $98 \pm 15$ & $123 \pm 12$ & $188 \pm 13$ & $1.3-2.3$ & $1.8 \pm 0.2$ \\
\hline MACSJ0600.1-2008 & 06:00:24.0-20:06:40.0 & 43 & $125 \pm 6$ & $286 \pm 15$ & $289 \pm 12$ & $165 \pm 13$ & $2.4-4.0$ & $3.3 \pm 0.1$ \\
\hline MACSJ2135.2-0102 ${ }^{C E}$ & $21: 35: 11.7-01: 02: 54.0$ & 57 & $120 \pm 9$ & $366 \pm 55^{i}$ & $325 \pm 49$ & $429 \pm 64$ & $1.9-3.2$ & $2.6 \pm 0.2$ \\
\hline
\end{tabular}

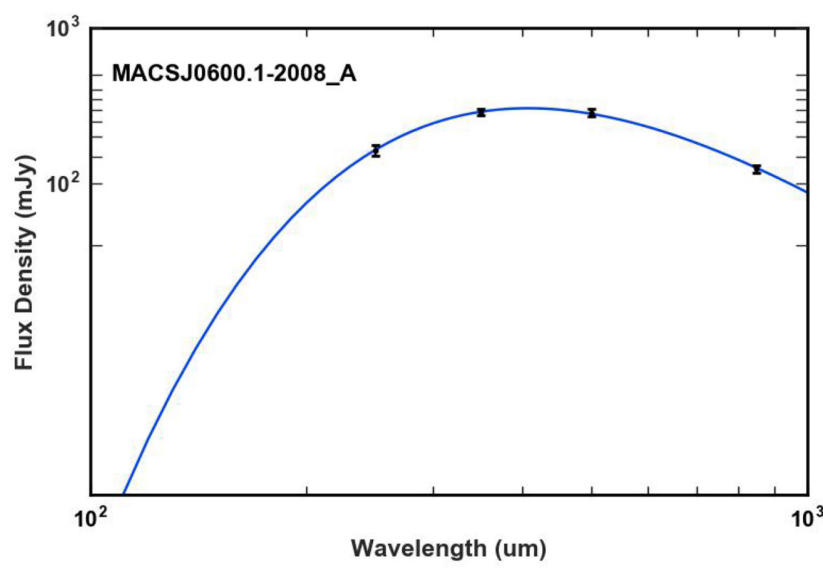

Figure 4. Greybody fits for the SPIRE 250-, 350-, 500- and SCUBA-2 850$\mu \mathrm{m}$ photometry for a fixed dust temperature of $40 \mathrm{~K}$. We show an example plot here; the remaining plots can be found in Appendix B in Fig. B1.

$\mathrm{S} / \mathrm{N} \geqslant 3.5$. Z15 concluded that the lines represent a multisource component with redshifts $z=2.04,3.25$, and 4.68. MACSJ1731 has a single line detection at $v=93.163 \mathrm{GHz}$, and $\mathrm{Z} 15$ deduced with follow-up observations that the line corresponds to $\mathrm{CO}(3-2)$, $z=2.712$. In all three cases where lines were detected, we find that the redshifts determined by Z15 fall within the photometric ranges deduced in this work. Furthermore the Cosmic Eyelash redshift of $z=2.3259$ also lies within the range quoted in Table 2. Following our AzTEC/RSR-S2CSS analysis, we have confidence that our redshift ranges are reliable for our other multiband detections in the survey.

\section{BRIGHTEST CLUSTER GALAXY PROPERTIES IN THE CENTIMETRE, MILLIMETRE AND SUBMILLIMETRE}

We have detected a number of BCGs with bright $850-\mu \mathrm{m}$ emission, and the nature of these sources was discussed in H15. However, because we have observed a large number of clusters, each containing a BCG, we can examine statistical 850- $\mu \mathrm{m}$ emission from the BCGs by stacking the SCUBA-2 maps at the optical positions of the BCGs (see Ebeling et al. 2001) in order to detect emission below the formal limit of an individual map. The presence of an AGN is an obvious contributor to the submillimetre emission of BCGs, and therefore, as an experiment, we also split the sample into groups according to the radio properties of the BCG. We excluded the submillimetre-bright detections from the main S2CSS as lensed galaxies which are bright in the submillimetre and found close to the BCG of the foreground cluster $(<0.1$ arcmin) would bias this analysis. By excluding these sources we can conduct an analysis of the average radio and submillimetre BCG properties without a contribution from potentially exceptionally bright lenses with $S_{850 \mu \mathrm{m}}>20 \mathrm{mJy}$. Furthermore, the Sunyaev-Zel'dovich effect (SZe; Sunyaev \& Zeldovich 1972) increment peak is at $\sim 850 \mu \mathrm{m}$ and may contaminate the central region (see Zemcov et al. 2010). However, because the S2CSS is a collection of filtered point sources and that the SZe is an extended emission component we can obtain a SZe-free thermal contribution at $350 \mathrm{GHz}$ by conducting an analysis on a complete stack of all our observations. 


\subsection{Radio data}

The NRAO VLA Sky Survey (NVSS; Condon et al. 1998) is a 1.4 $\mathrm{GHz}$ continuum survey at declinations greater than $-40^{\circ}$, with an angular resolution of 45 arcsec and a depth of $2.5 \mathrm{mJy}$ (Jones et al. 2015). The TGSS Alternative Data Release (TGSS-ADR; Intema et al. 2017) is a recent addition to the current TIFR GMRT Sky Survey (TGSS). The TGSS-ADR is a $147.5-\mathrm{MHz}$ radio survey covering a declination range of $-55^{\circ}$ to $+90^{\circ}$ to a median depth of 3 mJy with a 25-arcsec resolution. We matched the BCGs in each cluster to the NVSS and TGSS-ADR to detect radio-bright BCGs, identifying the radio sources within 3 arcsec of the optical position of the BCG. We found 105 matches for clusters targeted by the S2CSS.

\subsection{Stacking}

In order to stack sources, we binned the data by the NVSS $1.4-\mathrm{GHz}$ radio flux (because some BCGs will be radio-bright, containing an AGN, whereas others will be quiescent). Bins are distributed by splitting the matched data into interquartile-ranged (IQR) bins. The result is four bins that we classify as radio-faint (R-F), radiointermediate I (R-I), radio-intermediate II (R-II) and radio-bright (R-B), as described in Table 3. To evaluate the average $850-\mu \mathrm{m}$ flux density of the bins below the limit possible from our individual observations, we extracted thumbnail images from each SCUBA-2 map around the optical BCG location and evaluated the average flux density with inverse variance weighting, with the weight derived from an identical map extracted from the instrumental noise maps. We detected significant $850-\mu \mathrm{m}$ emission in the R-II and R-B bins, with $S_{850}=3.3 \pm 1.1$ and $S_{850}=3.8 \pm 1.1$ respectively, whereas the remaining bins are $<3 \sigma$. We constructed rest-frame SEDs for each of the four bins using the binned 1.4-GHz NVSS sources with their 140-MHz TGSS counterparts and the respective stacked 850- $\mu \mathrm{m}$ flux values. They are presented in Fig. 5.

The individual 1.4-GHz and 150-MHz sources were K-corrected using their respective spectroscopic cluster redshift. The SCUBA2 stacks were K-corrected to the median redshift of the bin. In Fig. 5. we show a fitted a power law of the form $S=A v^{\alpha}$ (solid line) using the least-squares algorithm in the SCIPY Python package simultaneously for the TGSS and NVSS data for each bin, the power law describes an expected synchrotron emission component of the cluster stack. We also show the power law calculated by the median of each individual spectral index within a bin (dotdashed line, median of set) for a comparison of methods. For an AGN, synchrotron emission would be the dominant feature, if star formation were to be the major contributor we would expect to see an excess in the 850- $\mu \mathrm{m}$ stacks compared with our extrapolated synchrotron power laws, this only likely in the R-B bin as the stack lays above the fitted power law.

In the two faintest bins, we found only upper limits for our $850-\mu \mathrm{m}$ stacks. The R-II bin $850-\mu \mathrm{m}$ stack lies below the fitted and median of the set of synchrotron power laws. The R-B bin displays a large variance; this is due to the nature of the bin, which contains extreme radio flux values with no upper constraint. The spectral indexes $(\alpha)$ approach a flatter state $(\alpha \rightarrow 0)$ in the first three bins, but owing to the large variance in the brightest bin, the trend turns over. However, in spite of the fact that the turnover is associated with the lack of constraints, there may well be a different mechanism involved, that powerful AGN or star formation are causing a spectrum shift.

\section{DISCUSSION}

From our sample, we identified a total of 105 sources with a bright submillimetre component in the field that are $>4 \sigma$, within 3.5 arcmin of the pointing, and exceed the ratio in equation (1). Of these 105 sources, eight are co-located with the BCG (confirmed by H15) and 18 have significant Herschel counterparts with four bands of IR $(250-850 \mu \mathrm{m})$ that are unlikely to belong to a cluster member. The remaining sources are candidate lenses as they have exceedingly bright corresponding IR luminosities, which would not be expected from blank fields because $N\left(S_{850 \mu \mathrm{m}}>15 \mathrm{mJy}\right)\left(\mathrm{deg}^{-2}\right) \approx 2$ (see Geach et al. 2017). A total of 97 ( $\sim 92$ per cent) of the reduced sample are candidate lensed SMGs $\left(S_{850}>15 \mathrm{mJy}\right)$ in the S2CSS sample.

Using these detections and our estimates of the sensitivity of each of the SCUBA-2 observations, we can determine the number of detections brighter than a certain $850-\mu \mathrm{m}$ flux expected per cluster surveyed to that depth. This calculation was made out to two different radii. The first was to within 1 arcmin of the $\mathrm{BCG}$, where the maps are most sensitive (see Fig. 2), and we assume that the maps reach a depth of $S_{850 \mu \mathrm{m}} / C_{80}>0.6$. The second was to within 3 arcmin of the BCG, where the map sensitivity is significantly lower and we can assume a depth of only $S_{850 \mu \mathrm{m}} / C_{80}>1.0$ over this full area in each cluster. Any very bright sources will be selected in any of our maps out to the 3-arcmin limit used, but for the faintest sources a significant contamination from spurious sources would be expected.

Fig. 6 shows the number counts derived for the S2CSS survey, split on whether a significant Herschel SPIRE counterpart to the SCUBA-2 source was found or not. The results show a significantly flatter slope to the counts for the central 1 arcmin, as would be expected for the strongly lensed sources detected in the core. It is also clear that there are very few $>5 \sigma$ sources without a SPIRE counterpart, particularly within 1 arcmin. The higher fraction of sources between $4<\sigma<5$ sources in this exclusively $850-\mu \mathrm{m}$ detected sample compared with those with a SPIRE counterpart suggests that more spurious sources are present. However, the possibility that at least some of these sources are at high redshift $(z$ $>4$ ) means that this sample deserves a careful follow-up.

We found that eight of the target clusters have a bright submillimetre component $\left(S_{850}>29 \mathrm{mJy}\right)$ co-located with their BCG. The most likely origin for the submillimetre emission in these massive, passive galaxies is from a central AGN. The submillimetre emission could arise from a synchrotron component, or alternatively from an advection-dominated accretion flow (ADAF; Mahadevan 1997), which contributes excess millimetre/submillimetre emission in addition to the synchrotron component; we note, however, that if there is strong synchrotron self-absorption then this could mimic millimetre excess emission. There could also be an alternative emission process, namely from remnant dust in the BCG heated by the stellar population; there may even be residual star formation present (Doi et al. 2005). Our BCG sample is discussed in H15, with follow-up AMI, CARMA and GISMO detections.

The brightest detection by far from this survey is MACSJ0600: with $S_{850 \mu \mathrm{m}}=125 \pm 7 \mathrm{mJy}$ and detected $\sim 4$ arcmin from the $\mathrm{BCG}$, it is a convincing candidate cluster-galaxy lensed SMG. MACSJ0600 was one of three selected targets having extreme SPIRE fluxes, and we added to the observed bands for this source with our SCUBA-2 data. Comparing MACSJ0600 source with all of the S2CSS observations observed in the MACS catalogue indicates that $<1$ per cent of the total number of clusters act as lenses for incredibly bright objects $\left(S_{850}>100 \mathrm{mJy}\right)$. Of the non-selected tar- 
Table 3. Each bin has been determined via interquartile sets from the 1.4-GHz data: the peak flux and error from each bin stack are presented here. We find that the radio-bright bin stack is also the bin with the highest significance $(3.3 \sigma)$ in the $850-\mu \mathrm{m}$ band. We show two spectral indexes: a fitted value based on fitting to the data, and a median of set values calculated by taking the median of the set of spectral indexes pooled from each of the clusters in the stack. Column 1, bin label (R-F, radio-faint; R-I and R-II, intermediate sample I and II; R-B, radio-bright); column 2, bin width; column 3, number of entries; column 4, mean 1.4-GHz flux with standard deviation error; column 5, peak stacked flux densities; column 6, fitted spectral index order; column 7, median set spectral index.

\begin{tabular}{|c|c|c|c|c|c|c|}
\hline Label & Bin & No. entries & $\begin{array}{c}\left\langle S_{1.4 \mathrm{GHz}}\right\rangle \\
{[\mathrm{mJy}]}\end{array}$ & $\begin{array}{c}\text { Peak } S_{850 \mu \mathrm{m}} \\
{[\mathrm{mJy}]}\end{array}$ & Fitted spectral index & Median set spectral index \\
\hline $\mathrm{R}-\mathrm{F}$ & $S_{1.4 \mathrm{GHz}} \leq 18 \mathrm{mJy}$ & 26 & $11 \pm 4$ & $2.7 \pm 1.1$ & $-0.99 \pm 0.01$ & $-0.91 \pm 0.05$ \\
\hline R-I & $18<S_{1.4 \mathrm{GHz}} \leq 41 \mathrm{mJy}$ & 26 & $27 \pm 6$ & $2.1 \pm 1.0$ & $-0.94 \pm 0.01$ & $-0.71 \pm 0.06$ \\
\hline $\mathrm{R}-\mathrm{B}$ & $S_{1.4 \mathrm{GHz}}>111 \mathrm{mJy}$ & 27 & $1981 \pm 468$ & $3.8 \pm 1.1$ & $-0.71 \pm 0.01$ & $-0.85 \pm 0.04$ \\
\hline
\end{tabular}

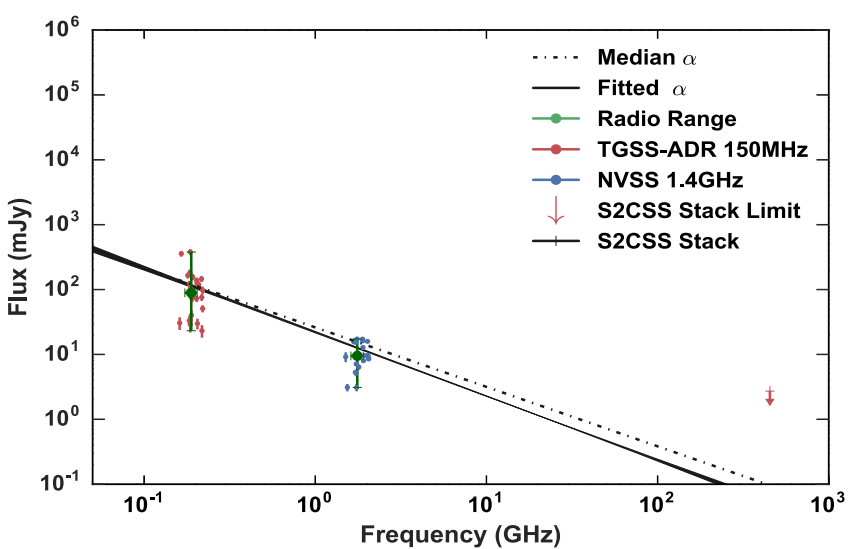

(a) Faint Sample (R-F): $\mathrm{S}_{1.4 \mathrm{GHz}} \leq 18 \mathrm{mJy}$

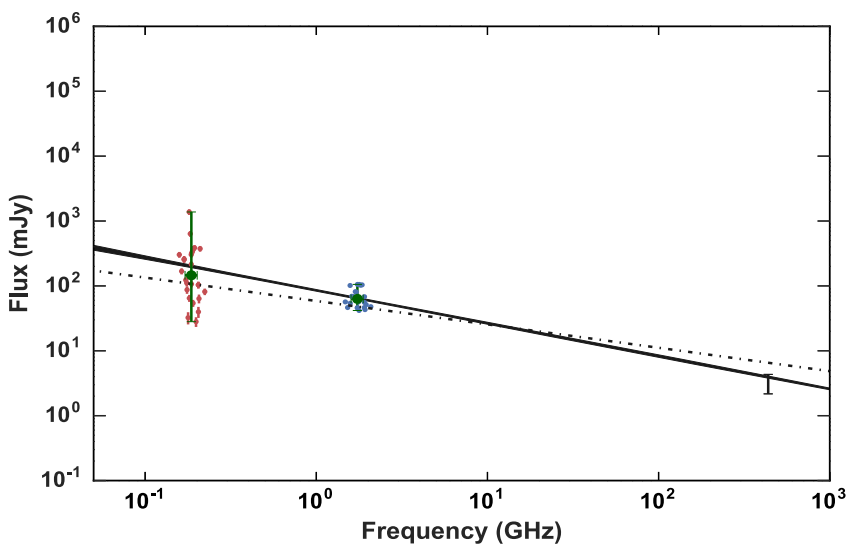

(c) Intermediate Sample (R-II): $41 \leq \mathrm{S}_{1.4 \mathrm{GHz}} \leq 111 \mathrm{mJy}$

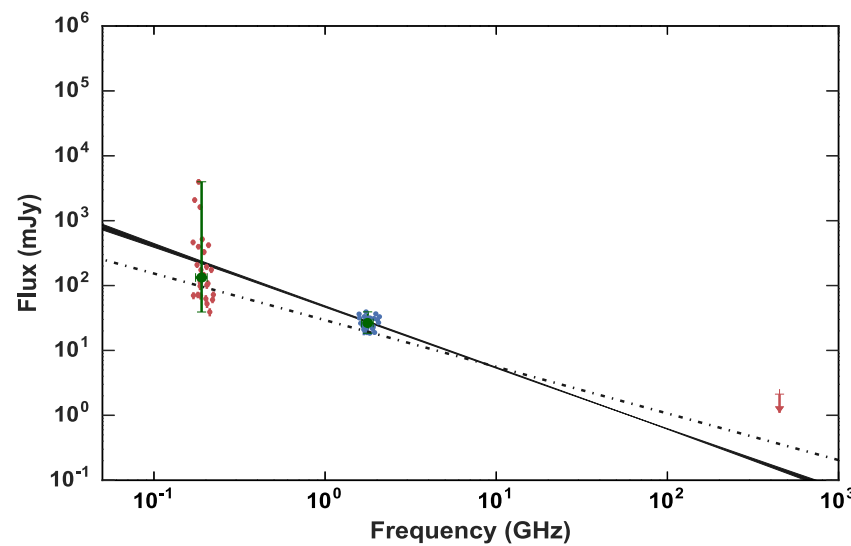

(b) Intermediate Sample (R-I): $18 \leq \mathrm{S}_{1.4 \mathrm{GHz}} \leq 41 \mathrm{mJy}$

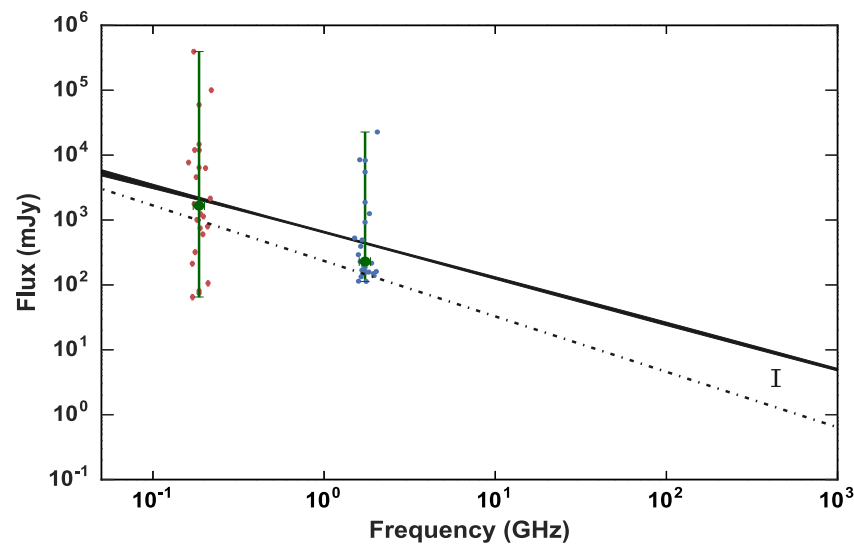

(d) Bright Sample (R-B): $\mathrm{S}_{1.4 \mathrm{GHz}}>111 \mathrm{mJy}$

Figure 5. The expected synchrotron emissions with $1 \sigma$ errors: red, TGSS-ADR; blue, NVSS; black, SCUBA-2; black solid line, power law of the form $S=$ $A \nu^{\alpha}$; black dot-dash line, median (a) The radio-faint (R-F) sample; (b) and (c) the intermediate selections I and II (R-I and R-II); (d) the radio-bright (R-B) sample. The overlaid bars show the median with the range in the flux values and the mean with the standard deviation in frequency. The stacks are binned by their 1.4-GHz data via interquartile ranging. The four stacks clearly show the change in the observed stacked 850- $\mu \mathrm{m}$ flux compared with the fitted power law. . This trend is suggestive of a greater contribution from the flatter spectrum core in brighter radio sources (R-B, R-II) compared with those in the fainter bins (R-I, R-F)

gets (excluding the Cosmic Eyelash), the brightest SCUBA-2 source is found in MACSJ1452.9+5802, with $S_{850 \mu \mathrm{m}}=98 \pm 18 \mathrm{mJy}$, and the brightest non-selected SCUBA-2 + SPIRE source was identified in MACSJ2043.2-2144, having $S_{850 \mu \mathrm{m}}=66 \pm 5 \mathrm{mJy}$.

BCGs tend to have gone through a number of mergers early in their evolution and to have already formed the bulk of their stars. Despite this, BCGs often still contain huge reservoirs of molecular gas, which should lead to easily observable star formation (e.g. Vantyghem et al. 2016). However, BCGs appear relatively passive and are generally considered to lack regions of significant star formation. There is growing evidence that this is not entirely true for a number of BCGs however, it may be that there are isolated starforming pockets driven and controlled through both AGN feedback and the 'cooling flow' mechanism (see Fabian 1994). Cooke et al. (2016) (C16) provide evidence of low star formation rates in BCGs for the redshift range $0.2<z<0.7$. They found that star formation does not contribute significantly to stellar mass growth, but that the radio emission in their sample is higher than that expected from star 

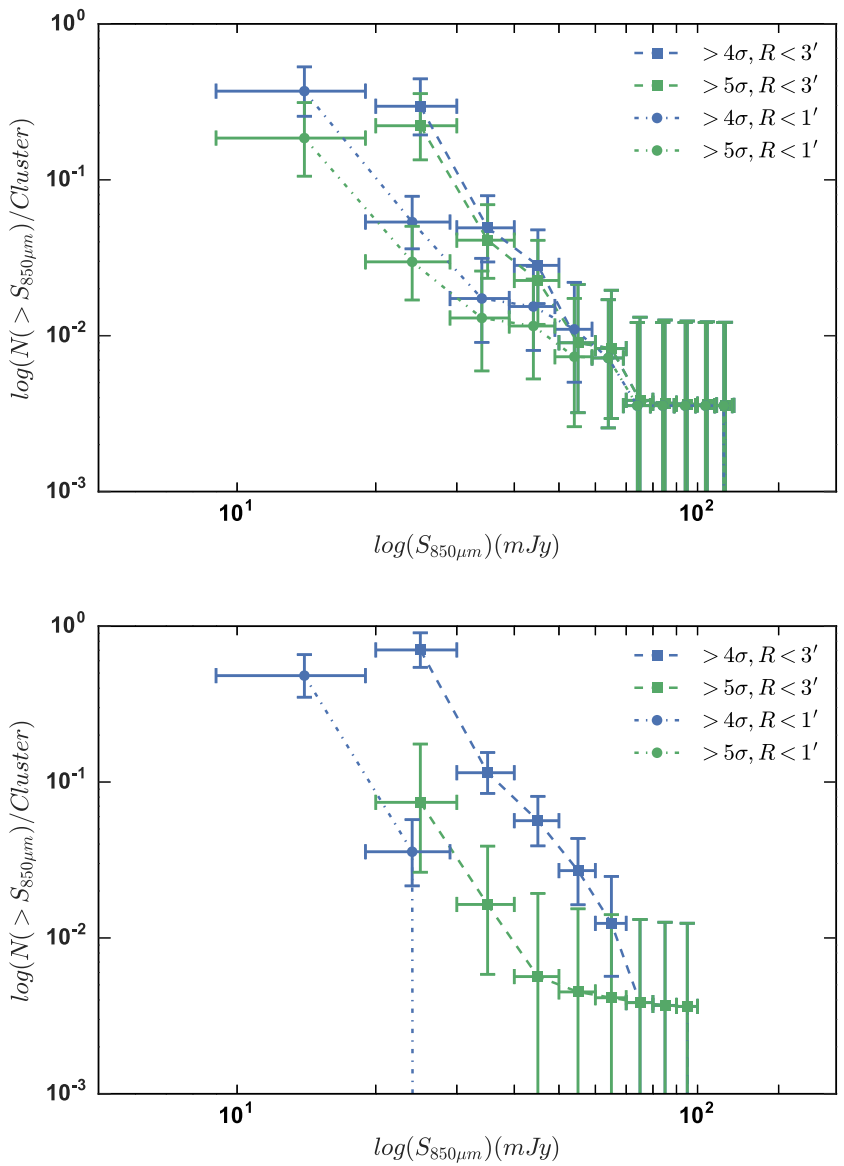

Figure 6. (Top) Number counts for the S2CSS sources, excluding BCGs, with a significant SPIRE counterpart within 1 or $3 \operatorname{arcmin}$ (solid or dashed lines) and according to detection significance ( $>5 \sigma$ green, $>4 \sigma$ blue). (Bottom) Number counts for SCUBA-2-only sources in the S2CSS catalogue. $R<1$-arcmin bins are centre-offset by -1 to disentangle the overlaps. Uncertainties in $850-\mu \mathrm{m}$ bins refer to the bin width $(5 \mathrm{mJy}$ in all cases), and the number counts are for a $1 \sigma$ confidence range assuming Poisson statistics (Gehrels 1986). No $>5 \sigma$ sources were found within 1 arcmin not having a bright SPIRE counterpart.

formation alone. It is likely that BCGs are dominated by AGNs in this waveband. McDonald et al. 2016 (M16) also looked at BCGs in the range $0.25<z<1.25$ and at their average properties. In the low-redshift range, $0.25<z<0.45$, the star formation rate is found to be $4-15 \mathrm{M}_{\odot} \mathrm{yr}^{-1}$, increasing with redshift as would be expected approaching the cosmic peak. Indeed, there may well be a hidden star formation contribution that has yet to be fully recognized and that may be hidden by powerful AGN activity.

Within clusters of galaxies, peak X-ray emissions coincide with the BCGs (Lin \& Mohr 2004), which may be indicative of cooling flows. These flows may be responsible for the observed star formation, but in the cases of more powerful AGNs in some BCGs, the star formation will not be as prominent in the radio and will be hidden by a dominant synchrotron component.

In this work, we have sought to further quantify the emissions by BCGs. We split our data into $1.4-\mathrm{GHz}$ bins and found that in increasing flux intensity radio bins the average $850-\mu \mathrm{m}$ signal also increases, both in intensity and in the signal-to-noise ratio. In the fainter bins, we see the upper limits of the 850- $\mu \mathrm{m}$ emission above the predicted emission based on the $1.4-\mathrm{GHz}$ and $140-\mathrm{MHz}$ observations, but as the average radio emission increases, so does the $850-\mu \mathrm{m}$ emission. We calculated the spectral index values in two ways: fitting and a median of the set. In the three faintest bins, the two methods provide similar results; in the brightest bin, the spectral index order deviates from a flattening spectrum trend significantly and occupies regions above and below the stack value, but this is probably because of the lack of an upper constraint to the bin. We conclude from our stacking analysis that the emission is primarily synchrotron, produced via an AGN in the central region, similar to the findings in $\mathrm{C} 16$ and M16. We note that this does not preclude ongoing active star formation in BCGs, but star formationmay well be being suppressed by a powerful AGN.

Identification of the origin of the $850-\mu \mathrm{m}$ emission at low redshifts $(z<1)$ is an important step for removing contamination from other large-scale millimetre/submillimetre surveys, for example when analysing the properties of the SZe. The SZe makes a strong contribution at $350 \mathrm{GHz}$, especially when observing clusters and the intra-cluster medium. Wide-field surveys seek to remove the SZ contribution by the removal of a contaminate map (e.g. Planck Collaboration et al. 2016). The advantage of the individual S2CSS maps is that each has been filtered as a point-source map in the reduction stage. Because the $\mathrm{SZe}$ is prominent on extended sources, filtering removes any potential SZe excess at $850 \mu \mathrm{m}$. In order to confirm this, we calculated the expected SZe contribution at $850 \mu \mathrm{m}$ for an average-size cluster $\left(M_{\mathrm{C}} \approx 10^{14} \mathrm{M}_{\odot}\right)$ to be $S_{\mathrm{SZe}}=0.03 \mathrm{mJy}$ (see Sunyaev \& Zeldovich 1980). Stacking all of the S2CSS maps provides an inverse variance average $850-\mu \mathrm{m}$ flux density of $S_{850 \mu \mathrm{m}}$ $=2.16 \pm 0.58 \mathrm{mJy}$. This is two orders of magnitude greater than the predicted SZe contribution.

\section{SUMMARY}

We have presented the first results from the SCUBA-2 Cluster Snapshot Survey, targeting a large number of massive clusters of galaxies in the poorest of submillimetre observing conditions in order to detect the brightest submillimetre sources. Our main findings can be summarized as follows.

(i) The SCUBA-2 Cluster Snapshot Survey took snapshots of 202 massive clusters, and we identified a total of $106850-\mu \mathrm{m}$ bright sources.

(ii) We identified 78 SCUBA-2 candidate lensed sources - these have no significant SPIRE detections and their IR luminosity is too great to be local. We identified eight sources that appear to be co-located with the BCG; these were reported previously by Hogan et al. (2015).

(iii) We identified 18 sources that were not only bright in the SCUBA-2 field but that also had exceptionally bright SPIRE counterparts (reported in Table 2).

(iv) We constructed basic photometric SEDs for 22 sources that have both SPIRE and SCUBA-2 detections (including the Cosmic Eyelash and three targeted sources). Encouragingly, in a precursor paper Zavala et al. (2015) undertook a spectroscopic analysis of four of these sources that lay within our photometric redshifts. Based on this agreement, we are confident that the ranges quoted for each source are reliable.

(v) We obtained radio fluxes for our sources from NVSS and TGSS-ADR, and constructed radio SEDs for various radio bins where we found a match (136 cases). Within four IQR bins, we stacked the corresponding SCUBA-2 imaging and found that the radio and $850-\mu \mathrm{m}$ emissions correlate with the radio luminosity, becoming increasingly more intense compared with the $850-\mu \mathrm{m}$ stacks. It is most probable that we are seeing intense AGN activity 
in the form of synchrotron emission, which may well be dominating low star formation in the brightest radio sources. However, as the radio intensity decreases we see low significant levels of a submillimetre excess, suggestive of ongoing star formation.

(vi) Finally, by stacking all of the observed clusters we were able to investigate the thermal contribution at $353 \mathrm{GHz}$ and to compare this with the expected SZ contribution. For a standard cluster with $M_{\mathrm{C}} \approx 10^{14} \mathrm{M}_{\odot}$, we found that the SZ contribution would be of order $S_{\mathrm{Sze}} \approx 0.03 \mathrm{mJy}$, whereas we found in stacking that $S_{850 \mu \mathrm{m}}=2.16 \pm 0.58 \mathrm{mJy}$ from lensed sources and BCGs. We conclude that the emissions in this waveband are dominated by thermal contributions and should be taken into careful consideration in millimetre/submillimetre surveys.

The S2CSS has produced a large number of lensed submillimetrebright sources by looking at X-ray-luminous clusters and we have investigated the submillimetre properties of a handful of BCGs. This survey contributes to the broader goal of hunting for more submillimetre-bright sources with the SCUBA-2 instrument. Further work will aim to make use of ALMA for high-resolution CO measurements of our potentially high-redshift sources and SCUBA2 follow-up observations.

\section{ACKNOWLEDGEMENTS}

RAC is supported by the Royal Society, and JEG is supported by a Royal Society University Research Fellowship. ACE acknowledges support from STFC grant ST/P00541/1. This research made use of the following software packages: SciPy (Jones et al. 2001), ASTROPY (Astropy Collaboration et al. 2013), MATPLOTLIB (Hunter 2007) and the KAPTEYN package (Terlouw \& Vogelaar 2015). IRS acknowledges support from an ERC Advanced Grant DUSTYGAL(321334), STFC(ST/P000J41/1) and a Royal Society/Wolfson Merit Award. The James Clerk Maxwell Telescope is operated by the East Asian Observatory on behalf of The National Astronomical Observatory of Japan, Academia Sinica Institute of Astronomy and Astrophysics, the Korea Astronomy and Space Science Institute, the National Astronomical Observatories of China and the Chinese Academy of Sciences (grant no. XDB09000000), with additional funding support from the Science and Technology Facilities Council of the United Kingdom and participating universities in the United Kingdom and Canada

\section{REFERENCES}

Aretxaga I. et al., 2007, MNRAS, 379, 1571

Astropy Collaboration et al., 2013, A\&A, 558, A33

Barger A. J., Cowie L. L., Sanders D. B., Fulton E., Taniguchi Y., Sato Y., Kawara K., Okuda H., 1998, Nature, 394, 248

Blain A. W., Longair M. S., 1996, MNRAS, 279, 847

Blain A. W., Smail I., Ivison R. J., Kneib J.-P., Frayer D. T., 2002, Phys. Rep., 369, 111

Bothwell M. S. et al., 2013, ApJ, 779, 67

Béthermin M. et al., 2015, A\&A, 573, A113

Böhringer H. et al., 2004, A\&A, 425, 367

Chapin E., Gibb A. G., Jenness T., Berry D. S., Scott D., Tilanus R. P. J., 2013, Starlink User Note, 258

Chapman S. C., Blain A. W., Smail I., Ivison R. J., 2005, ApJ, 622, 772

Combes F. et al., 2012, A\&A, 538, L4

Condon J. J., Cotton W. D., Greisen E. W., Yin Q. F., Perley R. A., Taylor G. B., Broderick J. J., 1998, AJ, 115, 1693

Conley A. et al., 2011, ApJ, 732, L35

Cooke K. C., O’Dea C. P., Baum S. A., Tremblay G. R., Cox I. G., Gladders M., 2016, ApJ, 833, 224
Coppin K. E. K. et al., 2011, MNRAS, 416, 680

Coppin K. et al., 2006, MNRAS, 372, 1621

Daddi E. et al., 2009, ApJ, 694, 1517

Danielson A. L. R. et al., 2011, MNRAS, 410, 1687

Danielson A. L. R. et al., 2013, MNRAS, 436, 2793

Danielson A. L. R. et al., 2017, ApJ, 840, 78

Datta R. et al., 2018, MNRAS, 2799

Decarli R. et al., 2012, ApJ, 752, 2

Dey A., Graham J. R., Ivison R. J., Smail I., Wright G. S., Liu M. C., 1999, ApJ, 519, 610

Doi A., Kameno S., Kohno K., Nakanishi K., Inoue M., 2005, MNRAS, 363,692

Draine B. T., 2006, ApJ, 636, 1114

Dye S. et al., 2008, MNRAS, 386, 1107

Eales S., Lilly S., Webb T., Dunne L., Gear W., Clements D., Yun M., 2000, AJ, 120, 2244

Ebeling H., Edge A. C., Bohringer H., Allen S. W., Crawford C. S., Fabian A. C., Voges W., Huchra J. P., 1998, MNRAS, 301, 881

Ebeling H., Edge A. C., Allen S. W., Crawford C. S., Fabian A. C., Huchra J. P., 2000, MNRAS, 318, 333

Ebeling H., Edge A. C., Henry J. P., 2001, ApJ, 553, 668

Ebeling H. et al., 2013, MNRAS,432, 62

Edge A. C., Ivison R. J., Smail I., Blain A. W., Kneib J. P., 1999, MNRAS, 306, 599

Edge A. C. et al., 2010, A\&A, 518, L47

Egami E. et al., 2010, A\&A, 518, L12

Fabian A. C., 1994, ARA\&A, 32, 277

Flewelling H. A. et al., 2016, preprint (arXiv:1612.05243)

Geach J. E. et al., 2013, MNRAS, 432, 53

Geach J. E. et al., 2017, MNRAS, 465, 1789

Gear W. K., Cunningham C. R., 1990, LIAC, 29, 353

Gehrels N., 1986, ApJ, 303, 336

George R. D. et al., 2014, MNRAS, 442, 1877

Hainline L. J., Blain A. W., Smail I., Alexander D. M., Armus L., Chapman S. C., Ivison R. J., 2011, ApJ, 740, 96

Hardcastle M. J. et al., 2013, MNRAS, 429, 2407

Hildebrand R. H., 1983, QJRAS, 24, 267

Hogan M. T. et al., 2015, MNRAS, 453, 1223

Holland W. S. et al., 1999, MNRAS, 303, 659

Holland W. S. et al., 2013, MNRAS, 430, 2513

Hopkins A. M., Beacom J. F., 2006, ApJ, 651, 142

Houck J. R., Schneider D. P., Danielson G. E., Neugebauer G., Soifer B. T., Beichman C. A., Lonsdale C. J., 1985, ApJ, 290, L5

Hughes D. H. et al., 1998, Nature, 394, 241

Hunter J. D., 2007, Comput. Sci. Eng., 9, 90

Intema H. T., Jagannathan P., Mooley K. P., Frail D. A., 2017, A\&A, 598, A78

Ivison R. J., Smail I., Le Borgne J.-F., Blain A. W., Kneib J.-P., Bezecourt J., Kerr T. H., Davies J. K., 1998, MNRAS,298, 583

Ivison R. J. et al., 2007, MNRAS, 380, 199

Ivison R. J. et al., 2010, A\&A, 518, L35

Jones E., , et al., 2001, SciPy

Jones S. F. et al., 2015, MNRAS, 448, 3325

Karim A. et al., 2013, MNRAS, 432, 2

Knudsen K. K., Watson D., Frayer D., Christensen L., Gallazzi A., Michałowski M. J., Richard J., Zavala J., 2017, MNRAS, 466, 138

Lilly S. J., Eales S. A., Gear W. K. P., Hammer F., Le Fèvre O., Crampton D., Bond J. R., Dunne L., 1999, ApJ, 518, 641

Lin Y.-T., Mohr J. J., 2004, ApJ, 617, 879

McDonald M. , 2016, ApJ, 817, 86

Madau P., Dickinson M., 2014, ARA\&A, 52, 415

Magnelli B. et al., 2012, A\&A, 539, A155

Mahadevan R., 1997, ApJ, 477, 585

Marrone D. P. et al., 2018, Nature, 553, 51

Nguyen H. T. et al., 2010, A\&A, 518, L5

Omont A. et al., 2013, A\&A, 551, A115

Orienti M., Dallacasa D., 2014, MNRAS, 438, 463

Ott S., 2010, ASPCS, 434, 139 
Planck Collaboration et al., 2014, A\&A, 571, A16

Planck Collaboration et al., 2016, A\&A, 594, A13

Rawle T. D. et al., 2012, ApJ, 747, 29

Saha P., 1995, AJ, 110, 916

Sanderson A. J. R., Edge A. C., Smith G. P., 2009, MNRAS, 398, 1698

Schneider P., Ehlers J., Falco E. E., 1992, Gravitational Lenses

Serjeant S., 2012, MNRAS, 424, 2429

Serjeant S. et al., 2008, MNRAS, 386, 1907

Simpson J. M. et al., 2014, ApJ, 788, 125

Simpson J. M. et al., 2015, ApJ, 807, 128

Smail I., Ivison R., Blain A., Kneib J.-P., 1999, AIPCS, 470, 312

Smail I., Ivison R. J., Blain A. W., 1997, ApJ, 490, L5

Smith G. P. et al., 2010, MNRAS, 409, 169

Spilker J. S. et al., 2016, ApJ, 826, 112

Sunyaev R. A., Zeldovich I. B., 1980, ARA\&A, 18, 537

Sunyaev R. A., Zeldovich Y. B., 1972, ASP, 4, 173
Swinbank A. M. et al., 2010, Nature, 464, 733

Tacconi L. J. et al., 2010, Nature, 463, 781

Terlouw J. P., Vogelaar M. G. R., 2015, Groningen

Thomas H. S., Currie M. J., 2014, Starlink Cookbook. p. 21.

Thomson A. P., Ivison R. J., Owen F. N., Danielson A. L. R., Swinbank A. M., Smail I., 2015, MNRAS, 448, 1874

Toft S. et al., 2014, ApJ, 782, 68

Tremblay G. R. et al., 2018, ApJ, 865, 13

Valtchanov I. et al., 2011, MNRAS, 415, 3473

Vantyghem A. N. et al., 2016, ApJ, 832, 148

Voges W. et al., 1999, A\&A, 349, 389

Zavala J. A. et al., 2015, MNRAS, 452, 1140

Zemcov M. et al., 2010, A\&A, 518 , 424

APPENDIX A: SURVEY SELECTION RESULTS.

Table A1. The 97 submillimetre sources detected from the given criteria. $C_{80}$ gives the 80 per cent completeness limit of the SCUBA-2 map, and uncertainties on flux densities are the $1 \sigma$ instrumental noise at the position of each detection peak. Column 1, map identification $(H$ indicates SPIRE counterparts, suffixes indicate multiple sources in the field, $C E$ indicates the Cosmic Eyelash, and $B C G$ indicates the bright cluster galaxy sources); column 2, peak location; column 3: angular separation between the candidate and cluster BCG; column 4, 80 per cent completeness; column 5 , peak 850 - $\mu \mathrm{m}$ flux density; column 6 , signal-to-noise ratio.

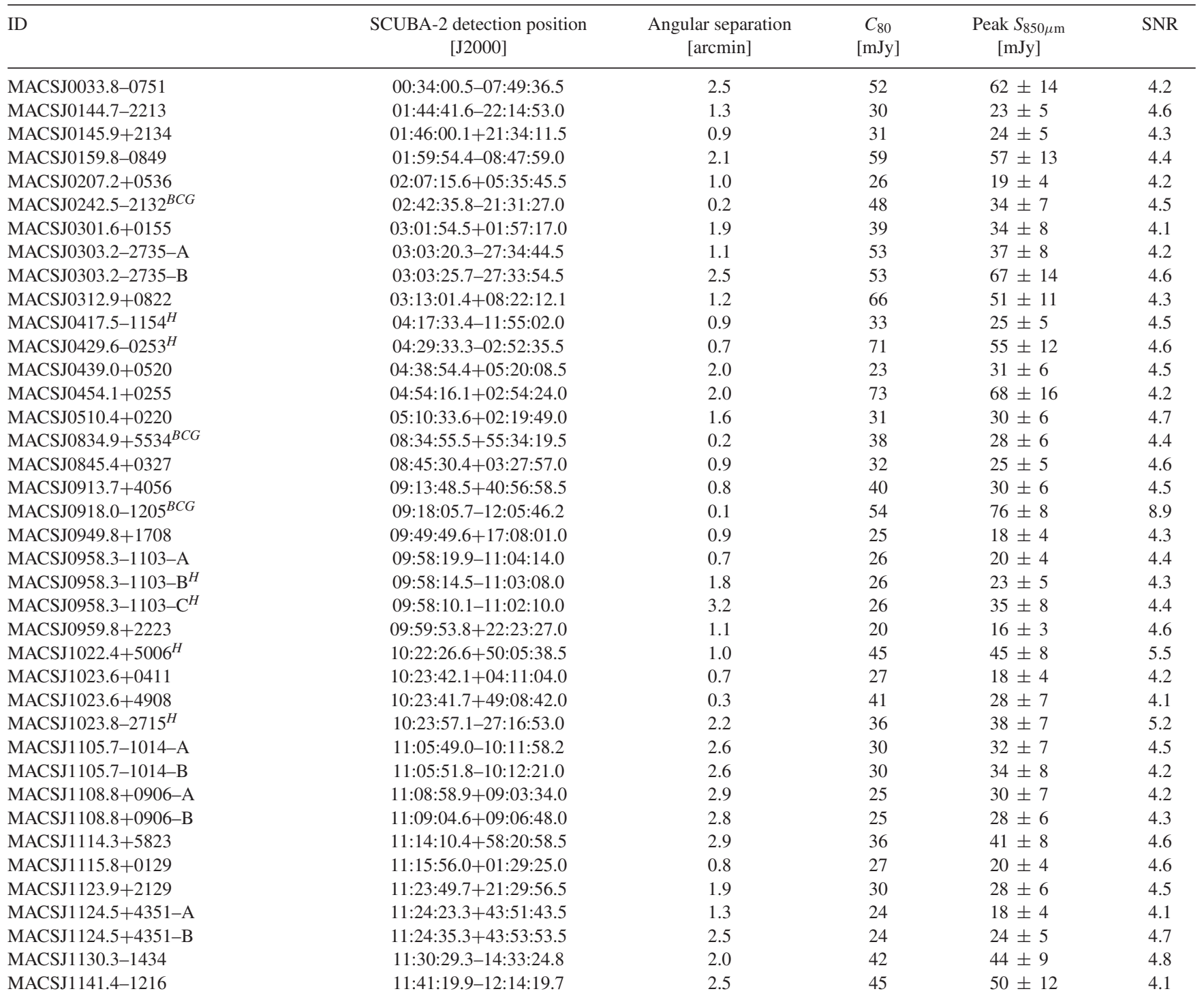


Table A1 - continued

\begin{tabular}{|c|c|c|c|c|c|}
\hline ID & $\begin{array}{l}\text { SCUBA-2 detection position } \\
\text { [J2000] }\end{array}$ & $\begin{array}{c}\text { Angular separation } \\
\text { [arcmin] }\end{array}$ & $\begin{array}{c}C_{80} \\
{[\mathrm{mJy}]}\end{array}$ & $\begin{array}{l}\text { Peak } S_{850 \mu \mathrm{m}} \\
\quad[\mathrm{mJy}]\end{array}$ & SNR \\
\hline MACSJ1157.3+3336 & $11: 57: 11.4+33: 37: 33.3$ & 1.5 & 25 & $19 \pm 4$ & 4.4 \\
\hline MACSJ1200.3+0320 ${ }^{H}$ & $12: 00: 15.2+03: 23: 17.5$ & 0.1 & 21 & $31 \pm 4$ & 6.7 \\
\hline MACSJ1203.2-2131 & $12: 03: 20.8-21: 32: 22.5$ & 1.8 & 28 & $21 \pm 5$ & 4.1 \\
\hline MACSJ1206.2-0847 & $12: 06: 18.7-08: 50: 29.0$ & 3.1 & 37 & $42 \pm 10$ & 4.1 \\
\hline MACSJ1218.4+4012 & $12: 18: 30.9+40: 11: 08.5$ & 1.8 & 38 & $35 \pm 7$ & 4.7 \\
\hline MACSJ1229.0+4737 ${ }^{H}$ & $12: 29: 09.1+47: 36: 50.3$ & 1.3 & 25 & $27 \pm 6$ & 4.5 \\
\hline MACSJ1259.3-0411 & $12: 59: 28.3-04: 10: 03.0$ & 2.2 & 33 & $40 \pm 7$ & 5.2 \\
\hline MACSJ1303.7-2415-A & $13: 03: 51.1-24: 16: 44.5$ & 2.4 & 52 & $55 \pm 12$ & 4.4 \\
\hline MACSJ1303.7-2415-B & $13: 03: 29.6-24: 14: 50.5$ & 3.2 & 52 & $63 \pm 13$ & 4.9 \\
\hline MACSJ1306.8+4633-A & $13: 06: 53.5+46: 30: 13.0$ & 3.0 & 26 & $28 \pm 6$ & 4.1 \\
\hline MACSJ1314.3-2515 & $13: 14: 21.2-25: 15: 47.5$ & 0.7 & 33 & $34 \pm 5$ & 6.1 \\
\hline MACSJ1314.4+6434 & $13: 14: 17.2+64: 33: 46.5$ & 1.2 & 31 & $21 \pm 5$ & 4.1 \\
\hline MACSJ1354.6+7715 & $13: 54: 41.9+77: 16: 41.5$ & 1.5 & 77 & $63 \pm 15$ & 4.2 \\
\hline MACSJ1359.1-1929 & $13: 59: 08.1-19: 26: 23.5$ & 2.7 & 55 & $58 \pm 14$ & 4.2 \\
\hline MACSJ1407.5-2701 ${ }^{B C G}$ & $14: 07: 29.8-27: 01: 04.2$ & 0.1 & 58 & $59 \pm 9$ & 6.5 \\
\hline MACSJ1411.3+5212-A & $14: 11: 28.2+52: 11: 18.0$ & 1.9 & 36 & $32 \pm 7$ & 4.4 \\
\hline MACSJ1411.3+5212-B & $14: 11: 27.1+52: 09: 44.0$ & 3.3 & 36 & $45 \pm 11$ & 4.0 \\
\hline MACSJ1447.4+0827 & $14: 47: 28.6+08: 27: 27.0$ & 0.8 & 38 & $27 \pm 6$ & 4.3 \\
\hline MACSJ1452.9+5802 & $14: 52: 44.4+58: 01: 35.5$ & 1.9 & 90 & $97 \pm 17$ & 5.5 \\
\hline MACSJ1500.3+2122 & $15: 00: 12.3+21: 21: 09.5$ & 2.2 & 51 & $50 \pm 9$ & 5.1 \\
\hline MACSJ1516.7+0701-A & $15: 16: 57.9+07: 01: 48.1$ & 3.4 & 49 & $60 \pm 14$ & 4.0 \\
\hline MACSJ1516.7+0701-B & $15: 16: 40.6+07: 02: 10.1$ & 1.3 & 49 & $34 \pm 8$ & 4.3 \\
\hline MACSJ1516.7+0701-C & $15: 16: 55.4+07: 01: 36.1$ & 2.7 & 49 & $54 \pm 12$ & 4.4 \\
\hline MACSJ1522.4+2742 & $15: 22: 27.7+27: 45: 38.5$ & 3.1 & 68 & $82 \pm 16$ & 5.1 \\
\hline MACSJ1651.1+0459 & $16: 51: 15.2+05: 00: 02.0$ & 1.9 & 33 & $32 \pm 6$ & 4.8 \\
\hline MACSJ1722.4+3207-A ${ }^{H}$ & $17: 22: 30.2+32: 07: 32.0$ & 1.0 & 39 & $26 \pm 5$ & 4.9 \\
\hline MACSJ1722.4+3207-B & $17: 22: 12.4+32: 08: 00.0$ & 3.0 & 39 & $50 \pm 9$ & 5.3 \\
\hline MACSJ1723.3+2350 & $17: 23: 17.2+23: 52: 03.0$ & 1.3 & 69 & $60 \pm 12$ & 4.8 \\
\hline MACSJ1727.3+5510-A & $17: 27: 35.0+55: 12: 23.8$ & 2.2 & 26 & $23 \pm 5$ & 4.4 \\
\hline MACSJ1727.3+5510-B $\mathrm{B}^{B C G}$ & $17: 27: 23.2+55: 10: 54.0$ & 0.1 & 26 & $34 \pm 4$ & 7.4 \\
\hline MACSJ1727.3+5510-C & $17: 27: 13.0+55: 11: 00.0$ & 1.5 & 26 & $22 \pm 5$ & 4.3 \\
\hline MACSJ1731.6+2252-A ${ }^{H}$ & $17: 31: 40.5+22: 50: 35.0$ & 2.2 & 30 & $48 \pm 5$ & 8.5 \\
\hline MACSJ1731.6+2252-B & $17: 31: 28.4+22: 52: 45.0$ & 2.7 & 30 & $35 \pm 8$ & 4.2 \\
\hline MACSJ1744.2+3259 & $17: 44: 14.3+32: 59: 27.4$ & 0.1 & 30 & $27 \pm 5$ & 5.5 \\
\hline MACSJ $1910.4+6741^{H}$ & $19: 10: 26.2+67: 41: 51.2$ & 0.4 & 37 & $27 \pm 6$ & 4.5 \\
\hline MACSJ2043.2-2144 ${ }^{H}$ & $20: 43: 14.5-21: 44: 37.0$ & 0.4 & 32 & $65 \pm 4$ & 13.5 \\
\hline MACSJ2050.7+0123-A ${ }^{H}$ & $20: 50: 38.0+01: 24: 58.5$ & 1.7 & 23 & $18 \pm 4$ & 4.4 \\
\hline MACSJ2050.7+0123-B & $20: 50: 45.5+01: 24: 54.5$ & 1.3 & 23 & $21 \pm 4$ & 4.8 \\
\hline MACSJ $2104.8+1401^{H}$ & $21: 05: 03.1+14: 00: 43.9$ & 2.6 & 46 & $52 \pm 12$ & 4.3 \\
\hline MACSJ2134.6-2706 & $21: 34: 24.4-27: 03: 49.5$ & 3.3 & 37 & $58 \pm 13$ & 4.5 \\
\hline MACSJ2135.2-0102 ${ }^{C E}$ & $21: 35: 11.7-01: 02: 54.0$ & 0.1 & 56 & $119 \pm 9$ & 13.1 \\
\hline MACSJ2153.6+1741 & $21: 53: 39.0+17: 39: 28.8$ & 2.4 & 39 & $36 \pm 7$ & 4.7 \\
\hline MACSJ2211.7-0349-A & $22: 11: 43.1-03: 50: 39.0$ & 1.0 & 22 & $16 \pm 3$ & 4.2 \\
\hline MACSJ2211.7-0349-B & $22: 11: 56.6-03: 48: 29.0$ & 3.3 & 22 & $40 \pm 9$ & 4.2 \\
\hline MACSJ2214.9-1359 & $22: 14: 57.8-14: 02: 24.0$ & 2.6 & 18 & $19 \pm 3$ & 4.8 \\
\hline MACSJ2258.6+2745-A & $22: 58: 38.3+27: 42: 49.5$ & 2.7 & 49 & $68 \pm 15$ & 4.4 \\
\hline MACSJ2258.6+2745-B & $22: 58: 32.7+27: 43: 05.5$ & 2.5 & 49 & $53 \pm 12$ & 4.4 \\
\hline MACSJ2325.3-1207 & $23: 25: 17.2-12: 08: 56.6$ & 1.6 & 46 & $36 \pm 8$ & 4.5 \\
\hline MACSJ2341.1+0018-A $\mathrm{A}^{B C G-H}$ & $23: 41: 07.0+00: 18: 34.5$ & 0.3 & 26 & $33 \pm 3$ & 8.4 \\
\hline
\end{tabular}




\section{APPENDIX B：REDSHIFT RANGES}
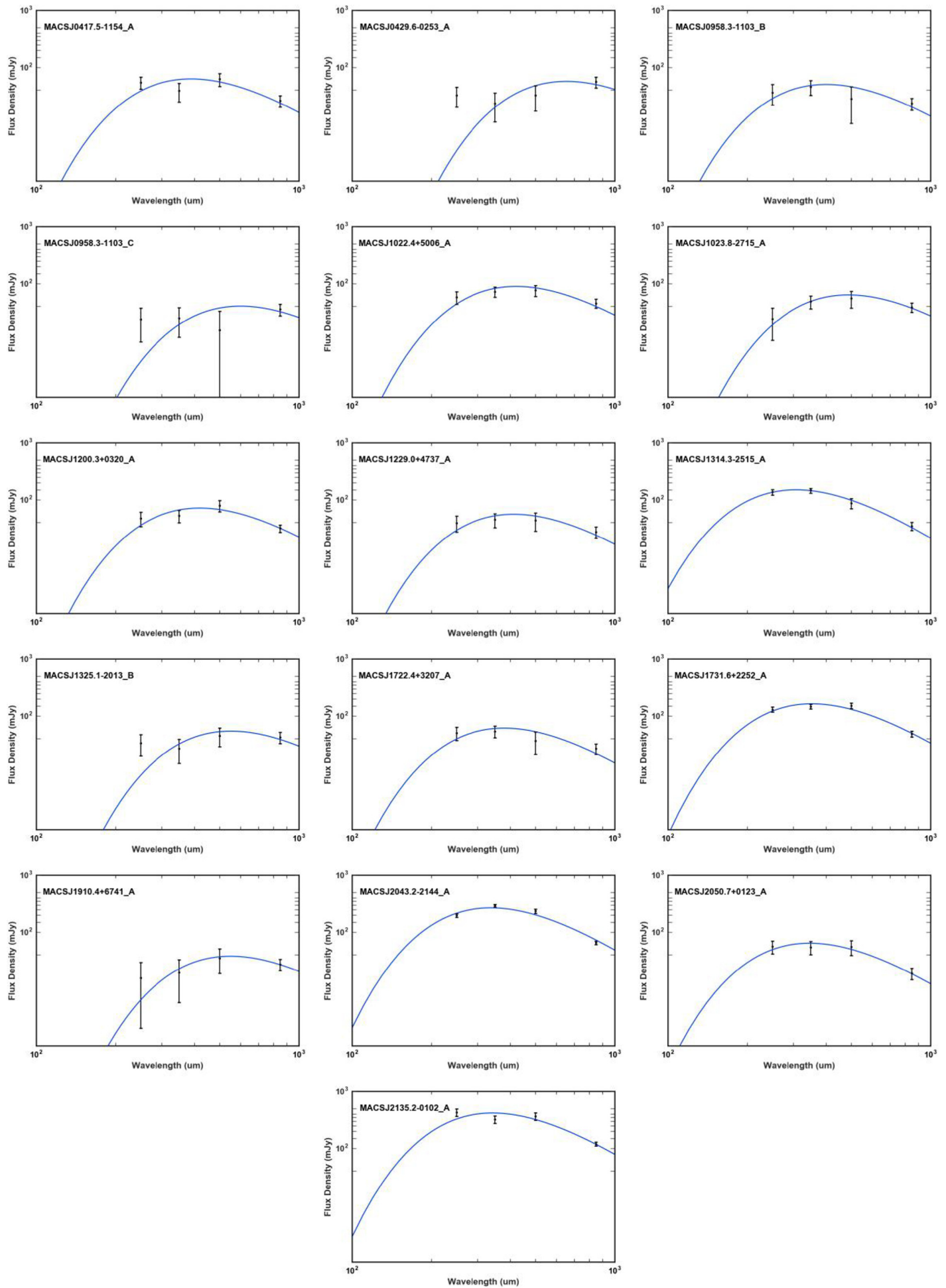

Figure B1. Greybodyfits for the SPIRE 250-, 350-, 500- and SCUBA-2 850- $\mu \mathrm{m}$ photometry for a fixed dust temperature of $40 \mathrm{~K}$. 

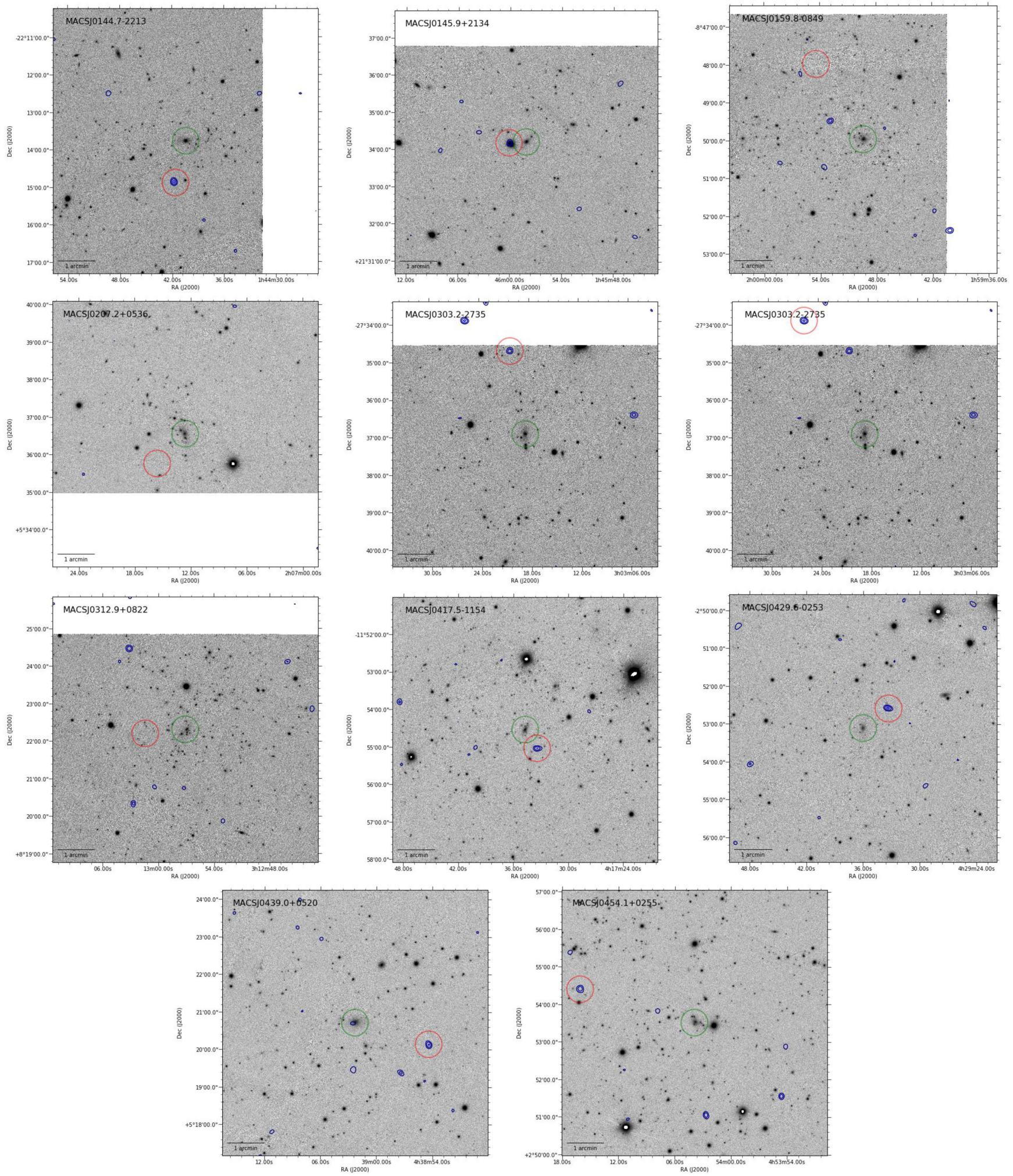

Figure C1. Pan-STARRSi-band imaging with SCUBA-2 contours overlaid ( $3 \sigma$ in $0.5 \sigma$ increments) of sources in Table A1. 

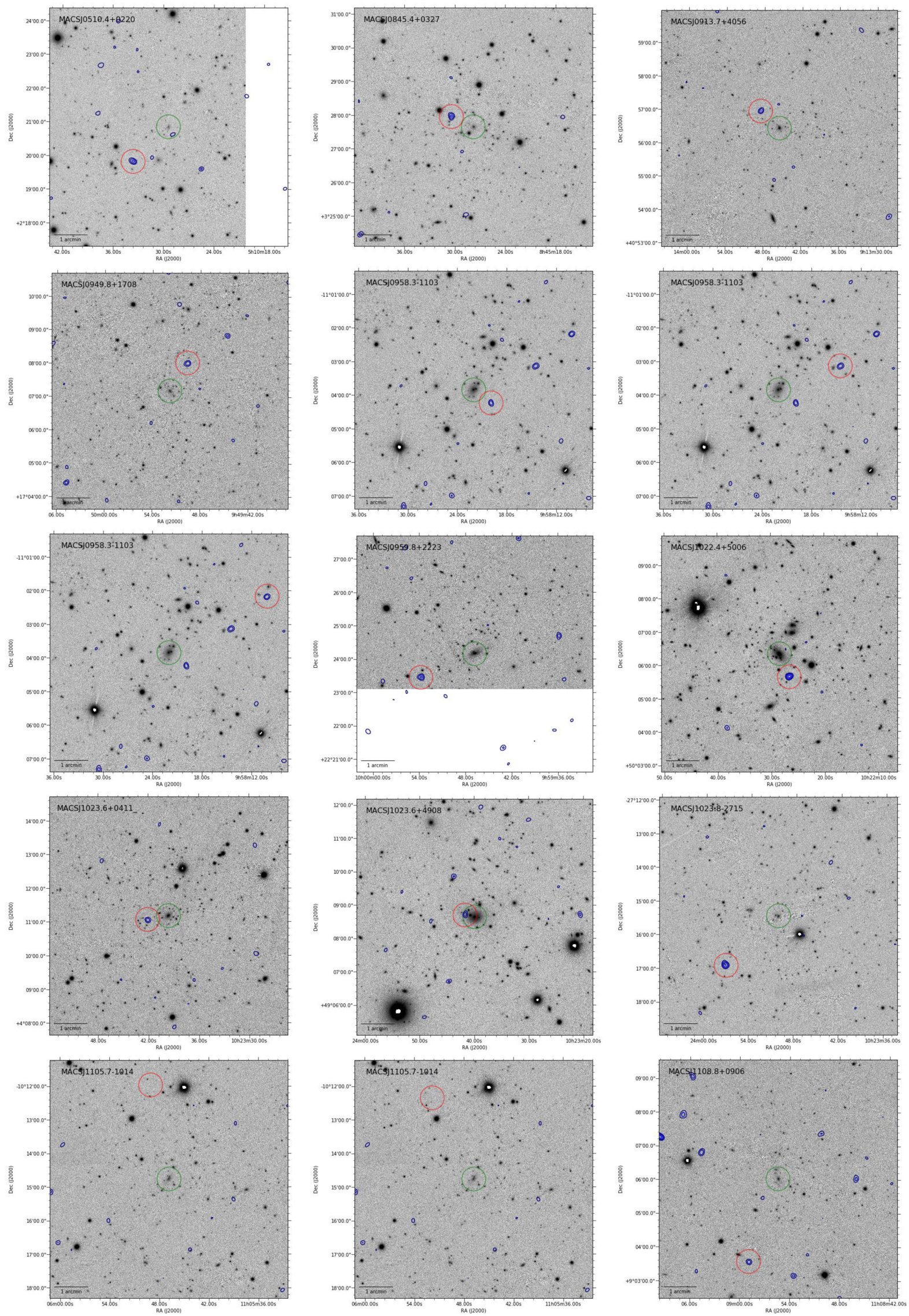

Figure C1 - Continued. 

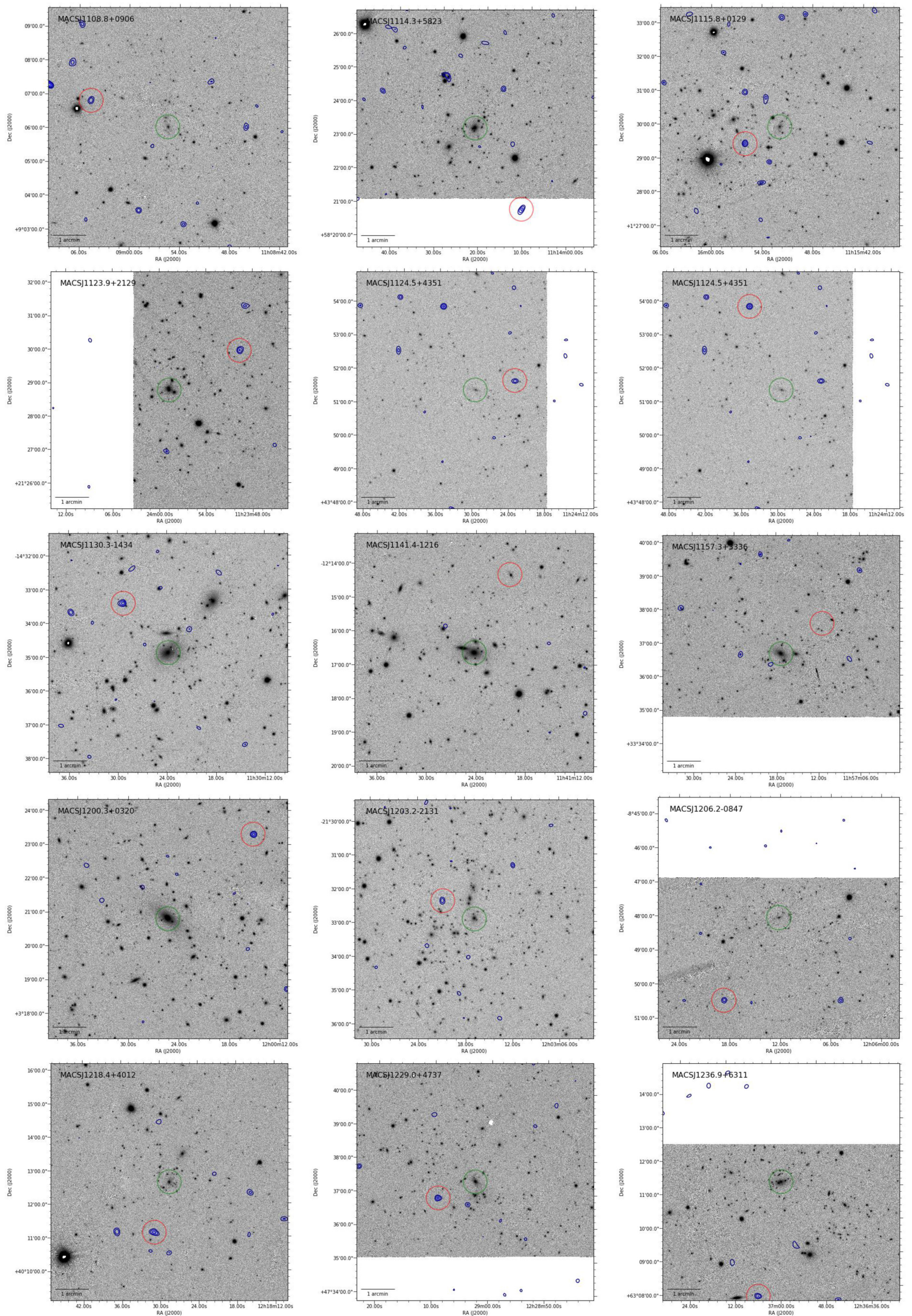

Figure C1 - Continued 

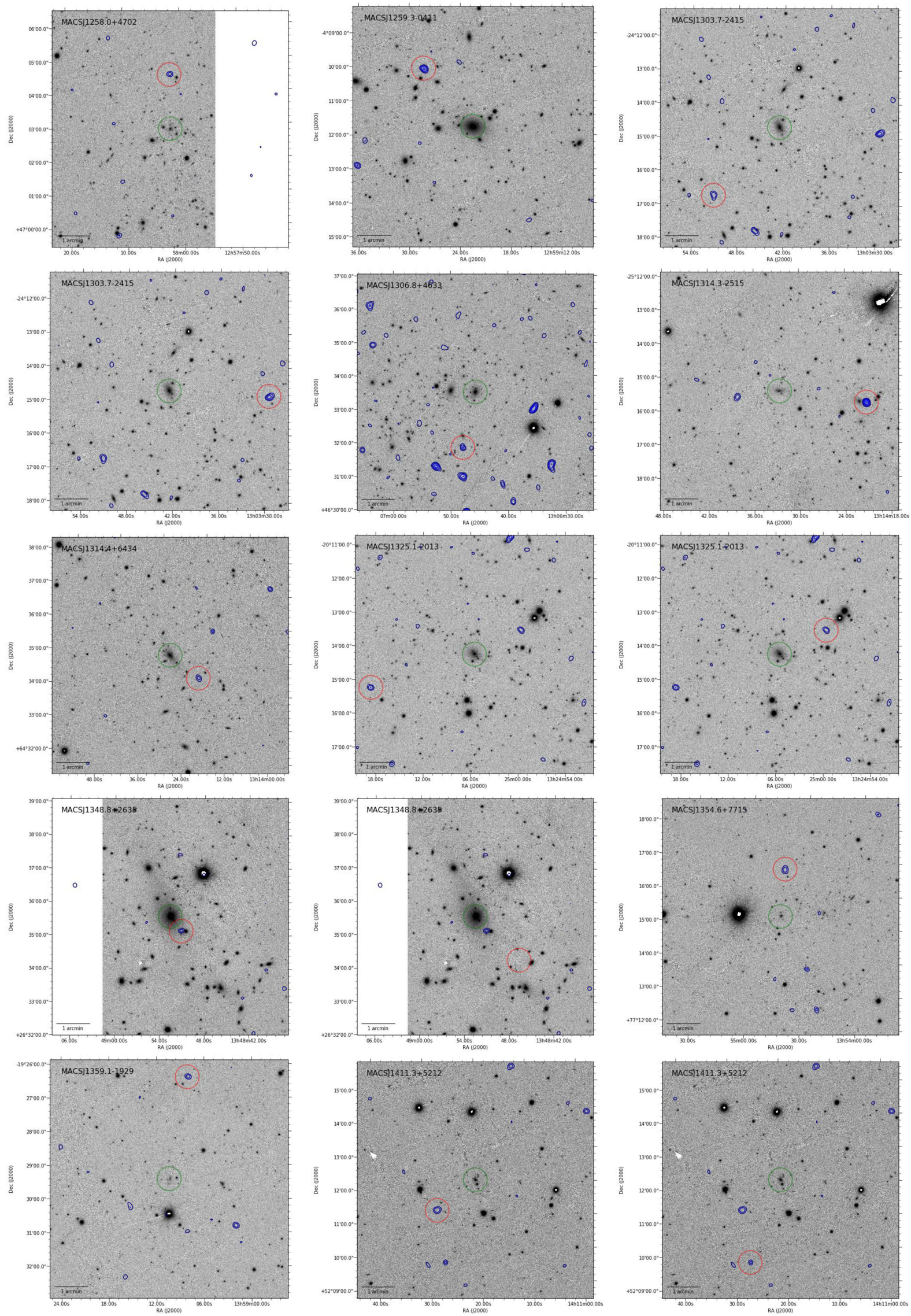

Figure C1 - Continued. 

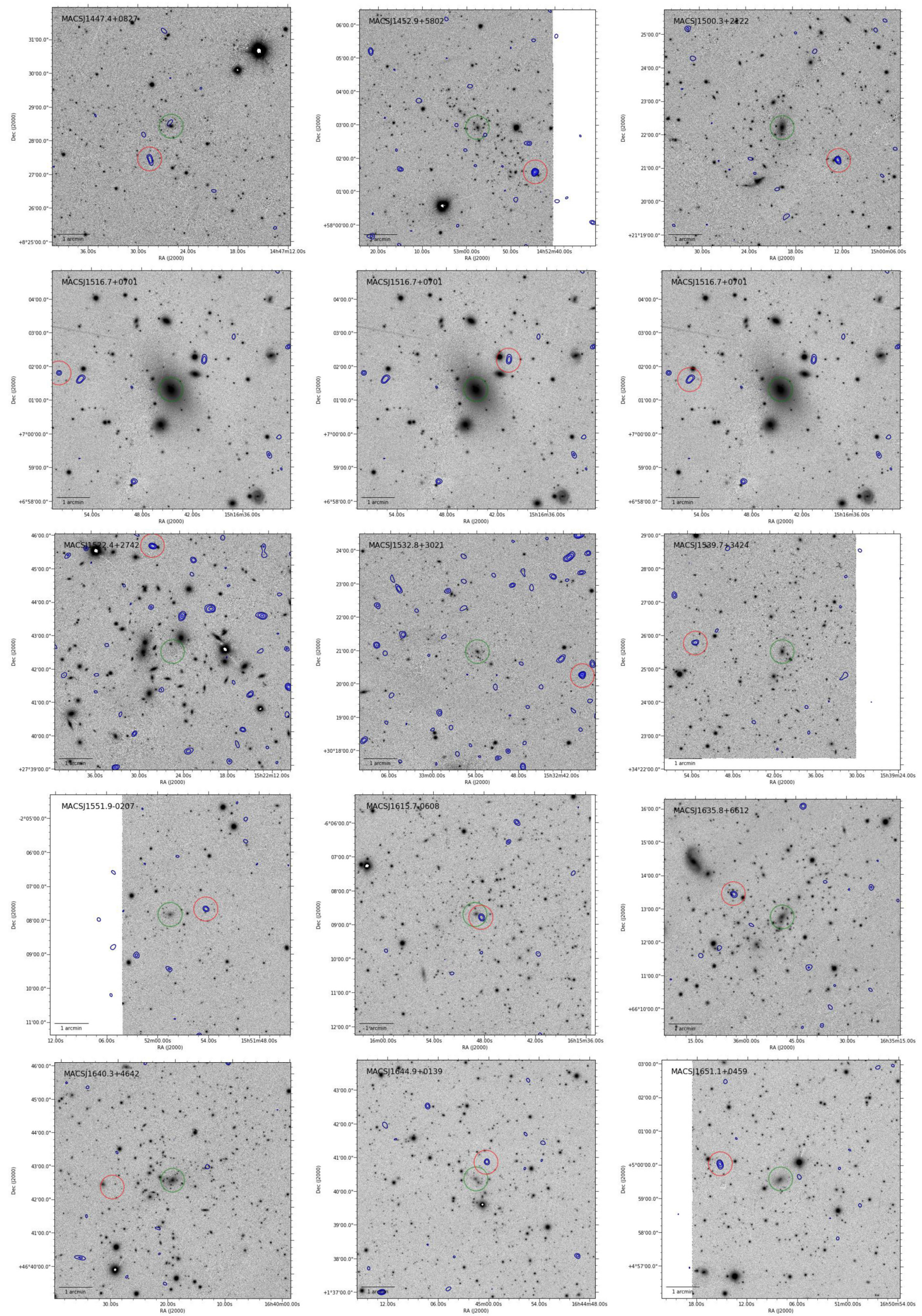

Figure C1 - Continued 

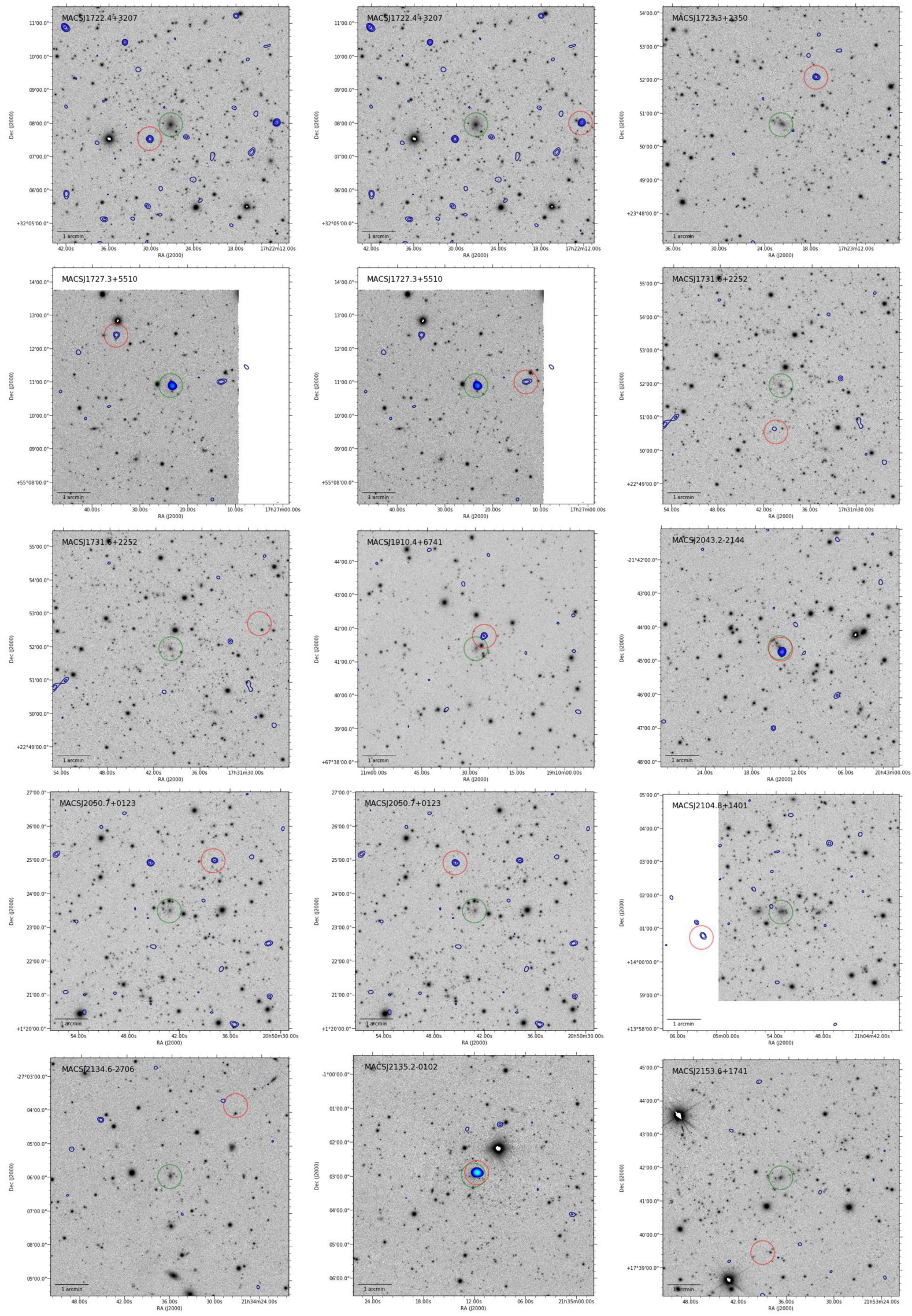

Figure C1 - Continued. 

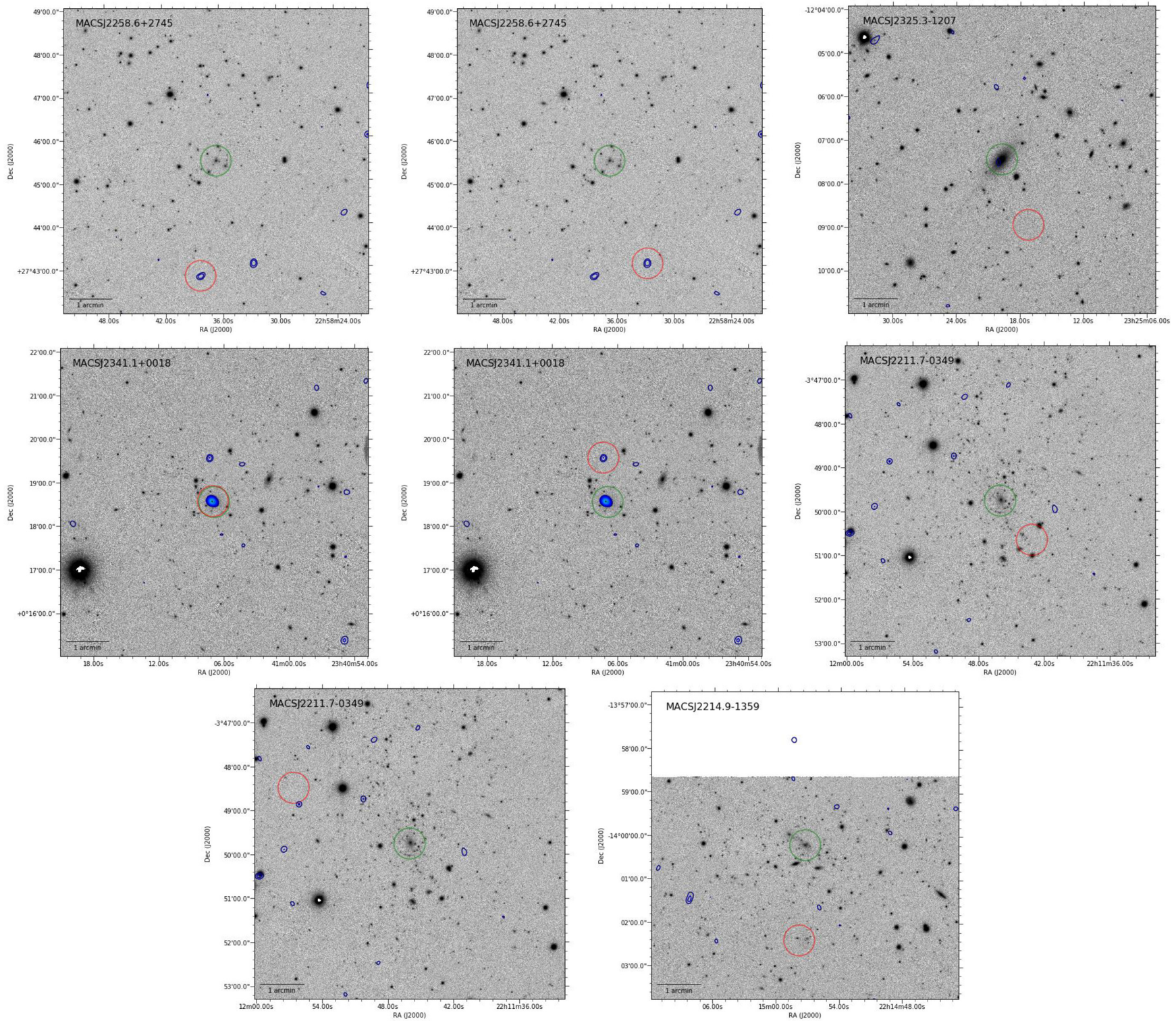

Figure C1 - Continued.

\section{APPENDIX D: TARGETED SOURCES}

A small number of sources were specifically targeted as follow-up to existing SPIRE detections. We observed MACSJ0326.8-0043, MACSJ0455.2+0657, MACSJ0600.1-2008 as part of the main survey, and their $S_{850 \mu \mathrm{m}}$ fluxes are given in Table D1. Fig. D1 shows the SCUBA-2 detection contours overlaid on Pan-STARRS $i$-band imaging. The SCUBA-2 observations were at the locations of these sources, but lay 3-4 arcmin from their host BCG and would not have been picked up if we had targeted the cluster BCG. Their redshift estimates for the fitted spectral energy distributions (determined in the same way as in Section 3.2) are included at the bottom of Table 2 .

Table D1. We list the three bright HLS sources that were targeted with the SCUBA-2 instrument for an additional submillimetre band. Column 1, cluster field; column 2, SCUBA-2 detection position; column 3, 80 per cent completeness sensitivity for the field; columns 4-7: submillimetre bands $250-850 \mu \mathrm{m}$.

\begin{tabular}{lccccrr}
\hline ID & $\begin{array}{c}\text { SCUBA-2 position } \\
{[\mathrm{J} 2000]}\end{array}$ & $\begin{array}{c}C_{80} \\
{[\mathrm{mJy}]}\end{array}$ & \multicolumn{1}{c}{$\begin{array}{c}S_{850} \\
{[\mathrm{mJy}]}\end{array}$} & \multicolumn{1}{c}{$\begin{array}{c}S_{250} \\
{[\mathrm{mJy}]}\end{array}$} & \multicolumn{1}{c}{\begin{tabular}{c}
\multicolumn{1}{c}{$S_{350}$} \\
{$[\mathrm{mJy}]$}
\end{tabular}} & \multicolumn{1}{c}{\begin{tabular}{c}
\multicolumn{1}{c}{$S_{500}$} \\
{$[\mathrm{mJy}]$}
\end{tabular}} \\
\hline MACSJ0326.8-0043 & $03: 26: 57.8-00: 45: 17$ & 36 & $29 \pm 6$ & $62 \pm 13$ & $73 \pm 13$ & $73 \pm 16$ \\
MACSJ0455.2+0657 & $04: 55: 18.1+07: 01: 01$ & 31 & $37 \pm 5$ & $189 \pm 14$ & $123 \pm 12$ & $98 \pm 16$ \\
MACSJ0600.1-2008 & $06: 00: 24.0-20: 06: 40$ & 44 & $125 \pm 7$ & $165 \pm 14$ & $290 \pm 12$ & $286 \pm 16$ \\
\hline
\end{tabular}



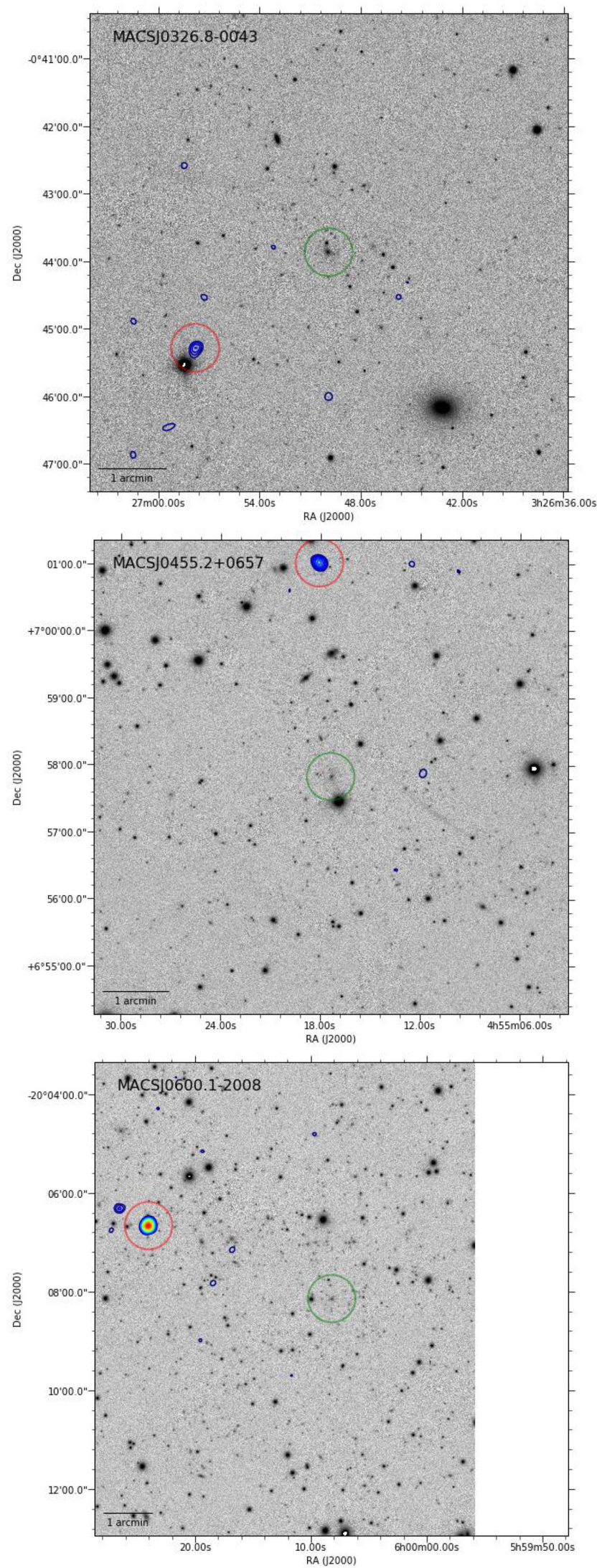

Figure D1. Pan-STARRS $i$-band imaging with SCUBA-2 contours overlaid ( $3 \sigma$ in $0.5 \sigma$ increments) of the three targeted sources from the S2CSS: MACSJ0326.8-0043, MACSJ0455.2+0657 and MACSJ0600.1-2008, red circles; BCGs, green circles. The displayed field for MACSJ0600.1-2008 is $r=4$ arcmin because of the location of the source.
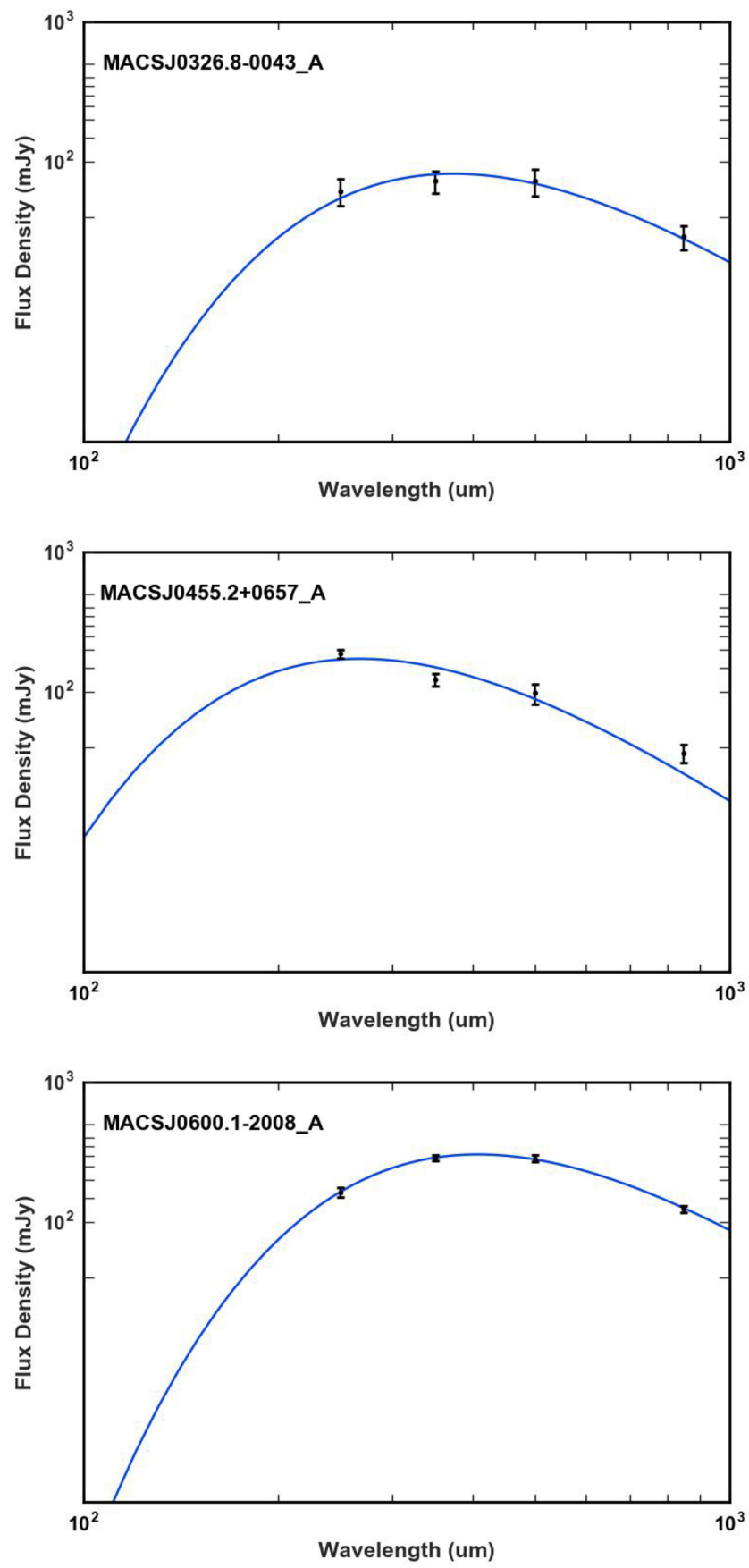

Figure D2. Greybody fits for the SPIRE 250-, 350-, 500- and SCUBA-2 $850-\mu \mathrm{m}$ photometry for a fixed dust temperature of $40 \mathrm{~K}$.

This paper has been typeset from a $\mathrm{T}_{\mathrm{E}} \mathrm{X} / \mathrm{L} \mathrm{T} \mathrm{E} \mathrm{X}$ file prepared by the author. 